\title{
Synthesis and Antiviral Evaluation of 6-(Alkyl-heteroaryl)furo[2,3- d]pyrimidin-2(3H)-one Nucleosides and Analogues with Ethynyl, Ethenyl, and Ethyl Spacers at C6 of the Furopyrimidine Core
}

\author{
Morris J. Robins, ' Ireneusz Nowak, Vivek K. Rajwanshi, Karl Miranda, John F. \\ Cannon, Matt A. Peterson, ${ }^{*}$ Graciela Andrei, Robert Snoeck, Erik De Clercq, ${ }^{\star}$ and \\ Jan Balzarini ${ }^{\ddagger}$ \\ Department of Chemistry and Biochemistry, Brigham Young University, Provo, Utah \\ 84602-5700, and Rega Institute for Medical Research, Katholieke Universiteit Leuven, B-3000 \\ Leuven, Belgium \\ morris_robins@byu.edu
}

\section{Supporting Information}

Pages S2-S22: Complete chemistry procedures and characterization data.

Page S23: Elemental analysis data.

Pages S24-S31: ${ }^{1} \mathrm{H}$ and ${ }^{13} \mathrm{C}$ NMR spectra of 15a-15c, 20a, 20c, 20d, 25a, and 25b.

Page S32: Supporting Information references. 


\section{Chemistry Experimental Section}

General. UV spectra were determined with solutions in $\mathrm{MeOH} .{ }^{1} \mathrm{H}(500 \mathrm{MHz})$ and ${ }^{13} \mathrm{C}(125$ $\mathrm{MHz}$ ) NMR spectra were recorded in $\mathrm{CDCl}_{3}$ unless otherwise noted. ${ }^{13} \mathrm{C}$ peaks with the same chemical shifts for more than one carbon are specified, and overlapping peaks for multiple carbons are indicated by a shift range (ovlp). HRMS were obtained with a Joel SX 102A double focusing mass spectrometer with an HP-9000 workstation. Elemental analyses were determined by M-H-W Laboratories, Phoenix, AZ. The (4-alkylphenyl)ethyne, 3-bromopyridine, 3butylpyridine, 3-bromo-6-iodopyridine, and 2-amino-5-bromopyrimidine starting materials were purchased from Aldrich. Compounds 5 and $\mathbf{6}$ were prepared as described, ${ }^{1}$ and $\mathbf{8 a}$ and $\mathbf{8 b}$ had spectroscopic data in agreement with reported values. ${ }^{2}$ General procedures $\mathrm{A}-\mathrm{C}$ were performed with the quantities and conditions noted for the individual compounds.

Procedure A (Synthesis of 6 and 9a). A solution of 5, the alkyne (4-5 equiv), $\left(\mathrm{Ph}_{3} \mathrm{P}\right)_{4} \mathrm{Pd}(0.1$ equiv), and $\mathrm{CuI}\left(0.2\right.$ equiv) in deoxygenated $\mathrm{DMF} / \mathrm{Et}_{3} \mathrm{~N}(2: 1, \mathrm{v} / \mathrm{v})$ was stirred under an inert atmosphere until the 6-bromofuro[2,3- $d]$ pyrimidin-2(3H)-one starting material had reacted completely (1-3 h, TLC). Volatiles were evaporated under reduced pressure, and the residue was purified by flash chromatography.

Procedure B (Deacetylation of 7a, 7a', 8a, 9a, and 10a). A solution of the O-acetyl nucleoside derivative in $\mathrm{NH}_{3} / \mathrm{MeOH}$ (saturated at $0{ }^{\circ} \mathrm{C}$ ) was stirred at $0{ }^{\circ} \mathrm{C}$ until deacetylation was complete (3-6 h, TLC). Volatiles were evaporated under reduced pressure, and the residue was purified by flash chromatagraphy.

Procedure C (Hydrogenation of 6 and 9). A mixture of 6 (or 9) and $10 \% \mathrm{Pd}-\mathrm{C}$ in $\mathrm{EtOH}$ (100\%) was shaken with $\mathrm{H}_{2}(25 \mathrm{psi})$ in a Parr apparatus at ambient temperature $(3-11 \mathrm{~h})$. The 
mixture was filtered (Celite), and the filter cake was washed with EtOH. Volatiles were evaporated under reduced pressure, and the residue was purified by flash chromatography.

(E/Z)-3-(3,5-Di-O-acetyl-2-deoxy- $\beta$-D-erythro-pentofuranosyl)-6-(dec-1-en-1-yl)furo[2,3-

d]pyrimidin-2(3H)-one (7a and 7a'). A mixture of 3-(3,5-di- $O$-acetyl-2-deoxy- $\beta$-D-erythropentofuranosyl)-6-(dec-1-yn-1-yl)furo[2,3-d]pyrimidin-2(3H)-one ${ }^{1}$ (6) (217 mg, $\left.0.46 \mathrm{mmol}\right)$, quinoline $(0.87 \mathrm{~mL}$, freshly distilled $)$, and Lindlar catalyst $(220 \mathrm{mg})$ in acetone $(60 \mathrm{~mL})$ was shaken with $\mathrm{H}_{2}$ (13 psi) at ambient temperature for $8 \mathrm{~h}$ in a Parr apparatus. [The reaction vessel was protected from light with aluminum foil to minimize isomerization; after $8 \mathrm{~h}$, a $500-\mathrm{MHz}{ }^{1} \mathrm{H}$ NMR spectrum indicated almost complete conversion of 6]. The suspension was filtered, the filter cake was washed with acetone $(100 \mathrm{~mL})$, and volatiles were evaporated from the combined filtrates. The residual yellow oil was flash chromatographed (EtOAc/hexanes, 1:1) to give 7a $(E / Z, 3: 7)$ as a light-yellow oil (142 mg, 65\%): UV max 347, 266, $228 \mathrm{~nm}$, min 308, $245 \mathrm{~nm} ;{ }^{1} \mathrm{H}$ NMR $\delta 8.24(\mathrm{~s}, 0.7 \mathrm{H}), 8.17(\mathrm{~s}, 0.3 \mathrm{H}), 6.52(\mathrm{dt}, J=7.4,15.2 \mathrm{~Hz}, 0.3 \mathrm{H}), 6.32(\mathrm{dd}, J=6.0,7.5 \mathrm{~Hz}$, $1 \mathrm{H}), 6.28(\mathrm{~s}, 1 \mathrm{H}), 6.18-6.10(\mathrm{~m}, 1 \mathrm{H}), 5.87(\mathrm{dt}, J=7.6,11.8 \mathrm{~Hz}, 0.7 \mathrm{H}), 5.23(\mathrm{~d}, J=6.0 \mathrm{~Hz}, 1 \mathrm{H})$, 4.43-4.39 (m, 3H), 2.99-2.94 (m, 1H), $2.53(\mathrm{ddd}, J=1.5,7.5,15.0 \mathrm{~Hz}, 1.4 \mathrm{H}), 2.25-2.19(\mathrm{~m}$, 0.6H), 2.12-2.05 (m, 1H), $2.12(\mathrm{~s}, 3 \mathrm{H}), 2.07(\mathrm{~s}, 3 \mathrm{H}), 1.49-1.45(\mathrm{~m}, 2 \mathrm{H}), 1.36-1.25(\mathrm{~m}, 10 \mathrm{H})$, 0.89-0.86 (m, 3H); ${ }^{13} \mathrm{C}$ NMR $\delta$ (172.0), 171.8, 170.6, 170.5, 155.7, (155.6), (154.74), 154.69, 139.1, (137.9), 134.4, (133.8), (117.0), 115.8, (108.6), 108.1, 101.4, (98.6), 88.7, (88.6), 83.40, (83.37), 74.29, (74.27), 63.9, 39.50, (39.46), (33.2), 32.0, 30.0, 29.70, 29.64, 29.59, 29.53, 29.44, $29.41,29.38,28.9,22.9,21.10,21.09,21.03,21.02,14.3$ (identifiable minor isomer peaks in parentheses); HRMS (EI) $m / z$ 474.2378 $\left(\mathrm{M}^{+}\left[\mathrm{C}_{25} \mathrm{H}_{34} \mathrm{~N}_{2} \mathrm{O}_{7}\right]=474.2366\right)$.

A solution of $7 \mathbf{a}(E / Z, 3: 7)(132 \mathrm{mg}, 0.28 \mathrm{mmol})$ in $\mathrm{CDCl}_{3}(5 \mathrm{~mL})$ was stirred for $6.5 \mathrm{~h}$ at $25-32{ }^{\circ} \mathrm{C}$ (water bath) with irradiation by a sun lamp $(250 \mathrm{~W}, 150 \mathrm{~V})$. Diastereoisomerization 
was monitored by ${ }^{1} \mathrm{H}$ NMR (500 MHz) until equilibrium was attained (7a'; E/Z, 9:1): UV $\max$ 339, 277, 251, $230 \mathrm{~nm}, \min$ 309, 269, $244 \mathrm{~nm} ;{ }^{1} \mathrm{H}$ NMR $\delta 8.24$ (s, 0.1H), 8.17 (s, 0.9H), 6.52 (dt, $J=7.3,15.8 \mathrm{~Hz}, 0.9 \mathrm{H}), 6.32(\mathrm{dd}, J=5.5,7.5 \mathrm{~Hz}, 1 \mathrm{H}), 6.28(\mathrm{~s}, 0.1 \mathrm{H}), 6.16(\mathrm{dt}, J=1.5,15.5 \mathrm{~Hz}$, $0.9 \mathrm{H}), 6.15(\mathrm{~s}, 0.9 \mathrm{H}), 6.13-6.11(\mathrm{~m}, 0.1 \mathrm{H}), 5.87(\mathrm{dt}, J=7.6,11.8 \mathrm{~Hz}, 0.1 \mathrm{H}), 5.23(\mathrm{~m}, 1 \mathrm{H})$, $4.41-4.39(\mathrm{~m}, 3 \mathrm{H}), 2.94(\mathrm{ddd}, J=2.4,5.6,14.6 \mathrm{~Hz}, 1 \mathrm{H}), 2.52(\mathrm{ddd}, J=1.5,7.5,15.0 \mathrm{~Hz}, 0.2 \mathrm{H})$, $2.23(\mathrm{ddd}, J=1.5,7.0,15.0 \mathrm{~Hz}, 1.8 \mathrm{H}), 2.15-2.06(\mathrm{~m}, 1 \mathrm{H}), 2.12(\mathrm{~s}, 3 \mathrm{H}), 2.06(\mathrm{~s}, 3 \mathrm{H}), 1.47$ (pent, $J=7.1 \mathrm{~Hz}, 2 \mathrm{H}), 1.34-1.25(\mathrm{~m}, 10 \mathrm{H}), 0.874(\mathrm{t}, J=7.3 \mathrm{~Hz}, 2.7 \mathrm{H}), 0.868(\mathrm{t}, J=7.1 \mathrm{~Hz}, 0.3 \mathrm{H}) ;{ }^{13} \mathrm{C}$ NMR $\delta 172.0,170.7,170.5,155.6,154.8,137.9,133.8,117.0,108.6,98.6,88.6,83.4,74.3$, $63.9,39.5,33.2,32.0,29.6,29.41,29.37,28.9,22.8,21.1,21.0,14.3$; HRMS (FAB) $\mathrm{m} / z$ 497.2269 ( $\left.\mathrm{M}+\mathrm{Na}^{+}\left[\mathrm{C}_{25} \mathrm{H}_{34} \mathrm{~N}_{2} \mathrm{O}_{7} \mathrm{Na}\right]=497.2264\right)$.

\section{(E)-6-(Dec-1-en-1-yl)-3-(2-deoxy- $\beta$-D-erythro-pentofuranosyl)furo[2,3- $d]$ pyrimidin-2(3H)-}

one (7b). Treatment of 7a' $(E / Z, 9: 1)(130 \mathrm{mg}, 0.27 \mathrm{mmol})$ with $\mathrm{NH}_{3} / \mathrm{MeOH}(20 \mathrm{~mL})$ by procedure $\mathrm{B}[4 \mathrm{~h}$, chromatography $(\mathrm{MeOH} / \mathrm{EtOAc}, 1: 20]$ gave $7 \mathbf{b}(E / Z, \sim 98: 2)$ as a white solid (57 mg, 53\%): UV max 346, 277, $226 \mathrm{~nm}(\varepsilon$ 9800, 13 700, 14000$), \min 309,244 \mathrm{~nm}(\varepsilon 4200,11$ 300); ${ }^{1} \mathrm{H}$ NMR (DMSO-d $) \delta 8.71(\mathrm{~s}, 1 \mathrm{H}), 6.56(\mathrm{~s}, 1 \mathrm{H}), 6.37-6.32(\mathrm{~m}, 2 \mathrm{H}), 6.15(\mathrm{t}, J=6.3 \mathrm{~Hz}$, $1 \mathrm{H}), 5.30(\mathrm{~d}, J=4.0 \mathrm{~Hz}, 1 \mathrm{H}), 5.14(\mathrm{t}, J=5.3 \mathrm{~Hz}, 1 \mathrm{H}), 4.24-4.21(\mathrm{~m}, 1 \mathrm{H}), 3.91(\mathrm{dd}, J=3.3,7.3$ $\mathrm{Hz}, 1 \mathrm{H}), 3.69-3.64(\mathrm{~m}, 1 \mathrm{H}), 3.64-3.58(\mathrm{~m}, 1 \mathrm{H}), 2.38$ (ddd, $J=4.0,5.8,13.5 \mathrm{~Hz}, 1 \mathrm{H}), 2.23-2.19$ $(\mathrm{m}, 2 \mathrm{H}), 2.05(\mathrm{dt}, J=6.5,13.0 \mathrm{~Hz}, 1 \mathrm{H}), 1.44-1.42(\mathrm{~m}, 2 \mathrm{H}), 1.28-1.25(\mathrm{~m}, 10 \mathrm{H}), 0.85(\mathrm{t}, J=6.8$ $\mathrm{Hz}, 3 \mathrm{H}) ;{ }^{13} \mathrm{C}$ NMR (DMSO- $\left.d_{6}\right) \delta 171.1,153.9,153.2,137.4,134.9,117.7,106.8,100.5,88.2$, 87.5, 69.7, 60.8, 41.2, 32.3, 31.3, 28.9, 28.7, 28.3, 22.1, 14.0; HRMS (FAB) $m / z 391.2216(\mathrm{M}+$ $\mathrm{H}^{+}\left[\mathrm{C}_{21} \mathrm{H}_{31} \mathrm{~N}_{2} \mathrm{O}_{5} \mathrm{Na}\right]=391.2233$ ). Anal. Calcd for $\mathrm{C}_{21} \mathrm{H}_{30} \mathrm{~N}_{2} \mathrm{O}_{5} \cdot 1.5 \mathrm{H}_{2} \mathrm{O}: \mathrm{C}, 60.83 ; \mathrm{H}, 8.07 ; \mathrm{N}$, 6.60. Found: C, 61.50; H, 7.67; N, 7.00. 


\section{3-(3,5-Di-O-acetyl-2-deoxy- $\beta$-D-erythro-pentofuranosyl)-6-decylfuro[2,3- $d]$ pyrimidin-}

2(3H)-one (8a). Treatment of 6 (89 mg, $0.19 \mathrm{mmol}), 10 \% \mathrm{Pd}-\mathrm{C}(10 \mathrm{mg})$, and EtOH (12 mL) by procedure $\mathrm{C}$ [3 h, chromatography (EtOAc/hexanes, 1:1 $\rightarrow$ 3:2)] gave 8a as a white solid (67 mg, 74\%): UV $\max 332,245 \mathrm{~nm}, \min 269,237 \mathrm{~nm} ;{ }^{1} \mathrm{H}$ NMR $\delta 8.16(\mathrm{~s}, 1 \mathrm{H}), 6.32(\mathrm{dd}, J=5.5$, $7.5 \mathrm{~Hz}, 1 \mathrm{H}), 6.11(\mathrm{~s}, 1 \mathrm{H}), 5.22(\mathrm{dt}, J=2.1,6.5 \mathrm{~Hz}, 1 \mathrm{H}), 4.41-4.38(\mathrm{~m}, 3 \mathrm{H}), 2.93(\mathrm{ddd}, J=2.1$, 5.4, 14.6 Hz, 1H), $2.64(\mathrm{t}, J=7.8 \mathrm{~Hz}, 2 \mathrm{H}), 2.11-2.05(\mathrm{~m}, 1 \mathrm{H}), 2.11(\mathrm{~s}, 3 \mathrm{H}), 2.08(\mathrm{~s}, 3 \mathrm{H}), 1.67$ (pent, $J=7.3 \mathrm{~Hz}, 2 \mathrm{H}), 1.36-1.25(\mathrm{~m}, 14 \mathrm{H}), 0.87(\mathrm{t}, J=7.3 \mathrm{~Hz}, 3 \mathrm{H}) ;{ }^{13} \mathrm{C} \mathrm{NMR} \delta 172.2,170.6$, $170.5,160.7,154.7,133.6,108.1,98.8,88.5,83.3,74.3,63.9,39.5,32.1,29.7,29.6,29.5,29.4$, 29.2, 28.5, 26.9, 22.8, 21.1, 21.0, 14.3; HRMS (FAB) $m / z 477.2599\left(\mathrm{M}+\mathrm{H}^{+}\left[\mathrm{C}_{25} \mathrm{H}_{37} \mathrm{~N}_{2} \mathrm{O}_{7}\right]=\right.$ 477.2602).

6-Decyl-3-(2-deoxy- $\beta$-D-erythro-pentofuranosyl)furo[2,3- $d]$ pyrimidin-2(3H)-one $(8 b)$.

Treatment of $8 \mathbf{a}(65 \mathrm{mg}, 0.14 \mathrm{mmol})$ with $\mathrm{NH}_{3} / \mathrm{MeOH}(10 \mathrm{~mL})$ by procedure $\mathrm{B}[3 \mathrm{~h}$, chromatography $(\mathrm{MeOH} / \mathrm{EtOAc}, 1: 30 \rightarrow 1: 15)]$ gave $\mathbf{8 b}$ as a white solid $(28 \mathrm{mg}, 51 \%)$ with spectral data as reported. ${ }^{2}$

\section{3-(3,5-Di- $O$-acetyl-2-deoxy- $\beta$-D-erythro-pentofuranosyl)-6-(phenylethynyl)furo[2,3-}

d]pyrimidin-2(3H)-one [9a(i)]. Treatment of $5(80 \mathrm{mg}, 0.19 \mathrm{mmol})$, phenylacetylene $(106 \mu \mathrm{L}$, $99 \mathrm{mg}, 0.97 \mathrm{mmol}),\left(\mathrm{Ph}_{3} \mathrm{P}\right)_{4} \mathrm{Pd}(22 \mathrm{mg}, 0.019 \mathrm{mmol}), \mathrm{CuI}\left(8 \mathrm{mg}, 0.04 \mathrm{mmol}^{2}, \mathrm{Et}_{3} \mathrm{~N}(1 \mathrm{~mL})\right.$, and DMF (2 mL), by general procedure A [1 h, chromatography (EtOAc/hexanes, 3:2)] gave 9a(i) (66 mg, 79\%) as an off-white solid: UV $\max 353,286,225 \mathrm{~nm}, \min 319,253 \mathrm{~nm} ;{ }^{1} \mathrm{H}$ NMR $\delta$ $8.38(\mathrm{~s}, 1 \mathrm{H}), 7.57-7.56(\mathrm{~m}, 2 \mathrm{H}), 7.42-7.37(\mathrm{~m}, 3 \mathrm{H}), 6.73(\mathrm{~s}, 1 \mathrm{H}), 6.31(\mathrm{dd}, J=5.8,7.8 \mathrm{~Hz}, 1 \mathrm{H})$, $5.24(\mathrm{~d}, J=6.5 \mathrm{~Hz}, 1 \mathrm{H}), 4.43-4.41(\mathrm{~m}, 3 \mathrm{H}), 2.99(\mathrm{ddd}, J=2.4,5.9,14.4 \mathrm{~Hz}, 1 \mathrm{H}), 2.14-2.09(\mathrm{~m}$, 1H), $2.13(\mathrm{~s}, 3 \mathrm{H}), 2.07(\mathrm{~s}, 3 \mathrm{H}) ;{ }^{13} \mathrm{C} \mathrm{NMR} \delta 171.4,170.6,170.5,154.6,139.2,135.9,132.0$, 
130.0, 128.8, 121.1, 108.3, 106.9, 97.9, 88.9, 83.6, 78.2, 74.2, 63.8, 39.5, 21.1, 21.0; HRMS (EI) $m / z 436.1287\left(\mathrm{M}^{+}\left[\mathrm{C}_{23} \mathrm{H}_{20} \mathrm{~N}_{2} \mathrm{O}_{7}\right]=436.1271\right)$.

\section{3-(2-Deoxy- $\beta$-D-erythro-pentofuranosyl)-6-(phenylethynyl)furo[2,3- $d]$ pyrimidin-2(3H)-}

one [9b(i)]. Treatment of $9 \mathbf{a}(\mathbf{i})(63 \mathrm{mg}, 0.14 \mathrm{mmol})$ with $\mathrm{NH}_{3} / \mathrm{MeOH}(14 \mathrm{~mL})$ by procedure $\mathrm{B}$ [5 h, chromatography $(\mathrm{MeOH} / \mathrm{EtOAc}, 1: 50 \rightarrow 1: 25)$ ] gave $\mathbf{9 b}(\mathbf{i})$ as a light-yellow solid $(41 \mathrm{mg}$, 83\%): UV $\max 353,285$ ( $\varepsilon 26000,25570)$, $\min 319,253 \mathrm{~nm}(\varepsilon 11190,10500) ;{ }^{1} \mathrm{H}$ NMR $\left(\mathrm{DMSO}_{6} \mathrm{~d}_{6}\right) \delta 8.90(\mathrm{~s}, 1 \mathrm{H}), 7.64-7.63(\mathrm{~m}, 2 \mathrm{H}), 7.51-7.47(\mathrm{~m}, 3 \mathrm{H}), 7.23(\mathrm{~s}, 1 \mathrm{H}), 6.14(\mathrm{t}, J=6.0$ $\mathrm{Hz}, 1 \mathrm{H}), 5.30(\mathrm{~d}, J=3.5 \mathrm{~Hz}, 1 \mathrm{H}), 5.15(\mathrm{t}, J=5.5 \mathrm{~Hz}, 1 \mathrm{H}), 4.24-4.21(\mathrm{~m}, 1 \mathrm{H}), 3.94(\mathrm{dd}, J=3.8$, $7.8 \mathrm{~Hz}, 1 \mathrm{H}), 3.67(\mathrm{dd}, J=3.8,12.3 \mathrm{~Hz}, 1 \mathrm{H}), 3.59(\mathrm{dd}, J=4.5,12.5 \mathrm{~Hz}, 1 \mathrm{H}), 2.43(\mathrm{ddd}, J=4.4$, 6.4, 13.4 Hz, 1H), $2.07(\mathrm{dt}, J=6.1,13.7 \mathrm{~Hz}, 1 \mathrm{H}) ;{ }^{13} \mathrm{C}$ NMR $\left(\mathrm{DMSO}-d_{6}\right) \delta 170.4,153.7,139.7$, 136.0, 131.5, 130.1, 129.0, 120.2, 110.7, 105.1, 96.5, 88.3, 87.9, 78.6, 69.6, 60.7, 41.2; HRMS (FAB) $m / z 375.0941\left(\mathrm{M}+\mathrm{Na}^{+}\left[\mathrm{C}_{19} \mathrm{H}_{16} \mathrm{~N}_{2} \mathrm{O}_{5} \mathrm{Na}\right]=375.0957\right)$. Anal. $\left(\mathrm{C}_{19} \mathrm{H}_{16} \mathrm{~N}_{2} \mathrm{O}_{5}\right) \mathrm{C}, \mathrm{H}, \mathrm{N}$.

\section{3-(3,5-Di-O-acetyl-2-deoxy- $\beta$-D-erythro-pentofuranosyl)-6-[(4-}

methylphenyl)ethynyl]furo[2,3-d]pyrimidin-2(3H)-one [9a(ii)]. Treatment of $\mathbf{5}(330 \mathrm{mg}$, $0.800 \mathrm{mmol})$, (4-methylphenyl)acetylene (0.52 mL, $470 \mathrm{mg}, 4.0 \mathrm{mmol}),\left(\mathrm{Ph}_{3} \mathrm{P}\right)_{4} \mathrm{Pd}(92 \mathrm{mg}, 0.08$ $\mathrm{mmol}), \mathrm{CuI}(30 \mathrm{mg}, 0.16 \mathrm{mmol}), \mathrm{Et}_{3} \mathrm{~N}(12.5 \mathrm{~mL})$, and $\mathrm{DMF}(25 \mathrm{~mL})$ by procedure A [ $3 \mathrm{~h}$, chromatography (EtOAc/hexanes, 3:2)] gave 9a(ii) (196 mg, 54\%) as a yellow solid: UV max 354, 291, $220 \mathrm{~nm}, \min 321,253 \mathrm{~nm} ;{ }^{1} \mathrm{H}$ NMR $\delta 8.36(\mathrm{~s}, 1 \mathrm{H}), 7.46(\mathrm{~d}, J=7.8 \mathrm{~Hz}, 2 \mathrm{H}), 7.20(\mathrm{~d}, J$ $=7.8 \mathrm{~Hz}, 2 \mathrm{H}), 6.70(\mathrm{~s}, 1 \mathrm{H}), 6.32(\mathrm{dd}, J=5.8,7.3 \mathrm{~Hz}, 1 \mathrm{H}), 5.24(\mathrm{~d}, J=6.5 \mathrm{~Hz}, 1 \mathrm{H}), 4.43-4.41$ (m, 3H), $3.00(\mathrm{ddd}, J=2.4,5.8,14.4 \mathrm{~Hz}, 1 \mathrm{H}), 2.40(\mathrm{~s}, 3 \mathrm{H}), 2.14-2.08(\mathrm{~m}, 1 \mathrm{H}), 2.13(\mathrm{~s}, 3 \mathrm{H})$, 2.07 (s, 3H); ${ }^{13} \mathrm{C}$ NMR $\delta 171.5,170.7,170.5,154.6,140.5,139.5,135.6,131.9,129.6,118.0$, 107.9, 107.1, 98.3, 88.9, 83.6, 77.7, 74.2, 63.9, 39.6, 21.9, 21.1, 21.0; HRMS (FAB) $\mathrm{m} / \mathrm{z}$ $451.1500\left(\mathrm{M}+\mathrm{H}^{+}\left[\mathrm{C}_{24} \mathrm{H}_{23} \mathrm{~N}_{2} \mathrm{O}_{7}\right]=451.1506\right)$. 


\section{3-(2-Deoxy- $\beta$-D-erythro-pentofuranosyl)-6-[(4-methylphenyl)ethynyl]furo[2,3-}

d]pyrimidin-2(3H)-one [9b(ii)]. Treatment of 9a(ii) (83 mg, $0.18 \mathrm{mmol})$ with $\mathrm{NH}_{3} / \mathrm{MeOH}(15$ $\mathrm{mL})$ by procedure $\mathrm{B}[3 \mathrm{~h}$, chromatography $(\mathrm{MeOH} / \mathrm{EtOAc}, 1: 30 \rightarrow 1: 10)]$ gave $\mathbf{9 b}(\mathbf{i i})$ as a yellow solid (50 mg, 74\%): UV max 354, $290 \mathrm{~nm}(\varepsilon 32$ 440, 34 400), $\min 320,252 \mathrm{~nm}(\varepsilon 14$ 740, 14 870); ${ }^{1} \mathrm{H}$ NMR (DMSO-d $)_{6} \delta 8.90(\mathrm{~s}, 1 \mathrm{H}), 7.51(\mathrm{~d}, J=8.3 \mathrm{~Hz}, 2 \mathrm{H}), 7.29(\mathrm{~d}, J=8.3 \mathrm{~Hz}$, 2H), $7.19(\mathrm{~s}, 1 \mathrm{H}), 6.14(\mathrm{t}, J=6.3 \mathrm{~Hz}, 1 \mathrm{H}), 5.30(\mathrm{~d}, J=4.5 \mathrm{~Hz}, 1 \mathrm{H}), 5.16(\mathrm{t}, J=5.0 \mathrm{~Hz}, 1 \mathrm{H})$, 4.25-4.21 (m, 1H), $3.94(\mathrm{dd}, J=4.0,7.5 \mathrm{~Hz}, 1 \mathrm{H}), 3.70-3.66(\mathrm{~m}, 1 \mathrm{H}), 3.64-3.60(\mathrm{~m}, 1 \mathrm{H}), 2.41$ (ddd, $J=4.3,6.1,13.6 \mathrm{~Hz}, 1 \mathrm{H}), 2.36(\mathrm{~s}, 3 \mathrm{H}), 2.09(\mathrm{dt}, J=6.1,13.3 \mathrm{~Hz}, 1 \mathrm{H}) ;{ }^{13} \mathrm{C}$ NMR (DMSO$\left.d_{6}\right) \delta 170.4,153.7,140.2,139.6,136.2,131.5,129.7,117.2,110.3,105.2,96.8,88.3,87.9,78.1$, 69.6, 60.7, 41.2, 21.2; HRMS (FAB) $m / z 367.1308\left(\mathrm{M}+\mathrm{H}^{+}\left[\mathrm{C}_{20} \mathrm{H}_{19} \mathrm{~N}_{2} \mathrm{O}_{5}\right]=367.1294\right)$. Anal. Calcd for $\mathrm{C}_{20} \mathrm{H}_{18} \mathrm{~N}_{2} \mathrm{O}_{5} \cdot 1.5 \mathrm{H}_{2} \mathrm{O}$ : C, 61.06; H, 5.38; N, 7.12. Found: C, 61.60; H, 4.92; N, 6.57.

\section{3-(3,5-Di-O-acetyl-2-deoxy- $\beta$-D-erythro-pentofuranosyl)-6-[(4-}

propylphenyl)ethynyl]furo[2,3- $\boldsymbol{d}]$ pyrimidin-2(3H)-one [9a(iii)]. Treatment of 5 (300 $\mathrm{mg}$, $0.722 \mathrm{mmol})$, (4-propylphenyl)acetylene (0.20 mL, $180 \mathrm{mg}, 1.25 \mathrm{mmol}),\left(\mathrm{Ph}_{3} \mathrm{P}\right)_{4} \mathrm{Pd}(84 \mathrm{mg}, 0.07$ $\mathrm{mmol}), \mathrm{CuI}(28 \mathrm{mg}, 0.15 \mathrm{mmol}), \mathrm{Et}_{3} \mathrm{~N}(7.5 \mathrm{~mL})$, and DMF $(15 \mathrm{~mL})$ by procedure $\mathrm{A}[3 \mathrm{~h}$, chromatography (EtOAc/hexanes, 3:2)] gave 9a(iii) $(227 \mathrm{mg}, 66 \%)$ as a yellow solid: UV max 352, 289, $225 \mathrm{~nm}, \min 322,260 \mathrm{~nm} ;{ }^{1} \mathrm{H}$ NMR $\delta 8.36(\mathrm{~s}, 1 \mathrm{H}), 7.47(\mathrm{~d}, J=8.3 \mathrm{~Hz}, 2 \mathrm{H}), 7.20(\mathrm{~d}, J$ $=8.3 \mathrm{~Hz}, 2 \mathrm{H}), 6.70(\mathrm{~s}, 1 \mathrm{H}), 6.32(\mathrm{dd}, J=5.5,7.5 \mathrm{~Hz}, 1 \mathrm{H}), 5.24(\mathrm{~d}, J=6.4 \mathrm{~Hz}, 1 \mathrm{H}), 4.43-4.41$ (m, 3H), $3.01(\mathrm{ddd}, J=2.4,5.8,14.4 \mathrm{~Hz}, 1 \mathrm{H}), 2.62(\mathrm{t}, J=7.7 \mathrm{~Hz}, 2 \mathrm{H}), 2.13(\mathrm{~s}, 3 \mathrm{H}), 2.13-2.08$ (m, 1H), $2.08(\mathrm{~s}, 3 \mathrm{H}), 1.66(\mathrm{sext}, J=7.7 \mathrm{~Hz}, 2 \mathrm{H}), 0.95(\mathrm{t}, J=7.5 \mathrm{~Hz}, 3 \mathrm{H}) ;{ }^{13} \mathrm{C}$ NMR $\delta 171.5$, $170.6,170.5,154.6,145.3,139.5,135.6,132.0,129.0,118.3,107.9,107.1,98.3,88.9,83.6,74.2$, 63.9, 39.6, 38.3, 24.5, 21.1, 21.0, 14.0; $\mathrm{HRMS}(\mathrm{FAB}) \mathrm{m} / z$ 501.1642 $\left(\mathrm{M}+\mathrm{Na}^{+}\left[\mathrm{C}_{26} \mathrm{H}_{26} \mathrm{~N}_{2} \mathrm{O}_{7} \mathrm{Na}\right]=\right.$ 501.1638). 


\section{3-(2-Deoxy- $\beta$-D-erythro-pentofuranosyl)-6-[(4-propylphenyl)ethynyl]furo[2,3-}

d]pyrimidin-2(3H)-one [9b(iii)]. Treatment of 9a(iii) (87 mg, $0.18 \mathrm{mmol})$ with $\mathrm{NH}_{3} / \mathrm{MeOH}(20$ $\mathrm{mL}$ ) by procedure $\mathrm{B}$ [3 h, chromatography (MeOH/EtOAc, 1:30)] gave $\mathbf{9 b}$ (iii) as a light-yellow solid (33 mg, 47\%): UV max 354, $291 \mathrm{~nm}$ (ع 17 800, 18 700), $\min 321,253 \mathrm{~nm}(\varepsilon$ 8100, 8200); ${ }^{1} \mathrm{H}$ NMR (DMSO- $\left.d_{6}\right) \delta 8.90(\mathrm{~s}, 1 \mathrm{H}), 7.53(\mathrm{~d}, J=8.3 \mathrm{~Hz}, 2 \mathrm{H}), 7.30(\mathrm{~d}, J=8.3 \mathrm{~Hz}, 2 \mathrm{H}), 7.20(\mathrm{~s}$, $1 \mathrm{H}), 6.14(\mathrm{t}, J=6.0 \mathrm{~Hz}, 1 \mathrm{H}), 5.30(\mathrm{~d}, J=4.5 \mathrm{~Hz}, 1 \mathrm{H}), 5.16(\mathrm{t}, J=5.3 \mathrm{~Hz}, 1 \mathrm{H}), 4.24-4.22(\mathrm{~m}$, $1 \mathrm{H}), 3.93(\mathrm{dd}, J=3.9,7.5 \mathrm{~Hz}, 1 \mathrm{H}), 3.68-3.66(\mathrm{~m}, 1 \mathrm{H}), 3.64-3.61(\mathrm{~m}, 1 \mathrm{H}), 2.60(\mathrm{t}, J=7.5 \mathrm{~Hz}$, 2H ), $2.42(\mathrm{ddd}, J=4.1,6.1,13.6 \mathrm{~Hz}, 1 \mathrm{H}), 2.09(\mathrm{dt}, J=6.1,13.3 \mathrm{~Hz}, 1 \mathrm{H}), 1.60$ (sext, $J=7.5 \mathrm{~Hz}$, 2H), $0.89(\mathrm{t}, J=7.3 \mathrm{~Hz}, 3 \mathrm{H}) ;{ }^{13} \mathrm{C}$ NMR $\left(\mathrm{DMSO}-d_{6}\right) \delta 170.5,153.8,144.8,139.6,136.2,131.5$, $129.1,117.5,110.3,105.2,96.8,88.3,87.9,78.1,69.6,60.7,41.2,37.1,23.8,13.6$; HRMS (FAB) $m / z$ 417.1425 $\left(\mathrm{M}+\mathrm{Na}^{+}\left[\mathrm{C}_{22} \mathrm{H}_{22} \mathrm{~N}_{2} \mathrm{O}_{5} \mathrm{Na}\right]=417.1426\right)$. Anal. Calcd for $\left(\mathrm{C}_{22} \mathrm{H}_{22} \mathrm{~N}_{2} \mathrm{O}_{5}\right) \cdot 0.5$ $\mathrm{H}_{2} \mathrm{O}: \mathrm{C}, 65.50 ; \mathrm{H}, 5.75 ; \mathrm{N}, 6.94$. Found: C, 66.01; H, 6.57; N, 6.33.

\section{3-(3,5-Di-O-acetyl-2-deoxy- $\beta$-D-erythro-pentofuranosyl)-6-[(4-}

pentylphenyl)ethynyl]furo[2,3- $d$ ]pyrimidin-2(3H)-one [9a(iv)]. Treatment of 5 (265 $\mathrm{mg}$, $0.638 \mathrm{mmol}),\left(4-\right.$ pentylphenyl)acetylene $(0.40 \mathrm{~mL}, 350 \mathrm{mg}, 2.1 \mathrm{mmol}),\left(\mathrm{Ph}_{3} \mathrm{P}\right)_{4} \mathrm{Pd}(74 \mathrm{mg}, 0.064$ $\mathrm{mmol}), \mathrm{CuI}(26 \mathrm{mg}, 0.14 \mathrm{mmol}), \mathrm{Et}_{3} \mathrm{~N}(8 \mathrm{~mL})$, and DMF $(16 \mathrm{~mL})$ by procedure A $[2 \mathrm{~h}$, chromatography (EtOAc/hexanes, 3:2)] gave 9a(iv) (160 mg, 49\%) as a yellow solid: UV max 355, 292, $225 \mathrm{~nm}, \min 321,254 \mathrm{~nm} ;{ }^{1} \mathrm{H}$ NMR $\delta 8.36(\mathrm{~s}, 1 \mathrm{H}), 7.47(\mathrm{~d}, J=8.3 \mathrm{~Hz}, 2 \mathrm{H}), 7.20(\mathrm{~d}, J$ $=8.3 \mathrm{~Hz}, 2 \mathrm{H}), 6.70(\mathrm{~s}, 1 \mathrm{H}), 6.32(\mathrm{dd}, J=5.5,7.5 \mathrm{~Hz}, 1 \mathrm{H}), 5.24(\mathrm{~d}, J=6.5 \mathrm{~Hz}, 1 \mathrm{H}), 4.43-4.41$ (m, 3H), $3.00(\mathrm{ddd}, J=2.4,5.8,14.4 \mathrm{~Hz}, 1 \mathrm{H}), 2.63(\mathrm{t}, J=7.8 \mathrm{~Hz}, 2 \mathrm{H}), 2.14-2.08(\mathrm{~m}, 1 \mathrm{H}), 2.13$ $(\mathrm{s}, 3 \mathrm{H}), 2.08(\mathrm{~s}, 3 \mathrm{H}), 1.65-1.61(\mathrm{~m}, 2 \mathrm{H}), 1.36-1.31(\mathrm{~m}, 4 \mathrm{H}), 0.90(\mathrm{t}, J=6.8 \mathrm{~Hz}, 3 \mathrm{H}) ;{ }^{13} \mathrm{C} \mathrm{NMR} \delta$ $171.5,170.6,170.5,154.6,145.5,139.5,135.6,132.0,130.4,128.9,118.2,107.9,107.1,98.4$ 
$88.9,83.6,77.7,74.2,63.9,39.6,36.2,31.6,31.0,22.7,21.1,21.0,14.2$; HRMS (FAB) $\mathrm{m} / z$ $529.1949\left(\mathrm{M}+\mathrm{Na}^{+}\left[\mathrm{C}_{28} \mathrm{H}_{30} \mathrm{~N}_{2} \mathrm{O}_{7} \mathrm{Na}\right]=529.1951\right)$.

\section{3-(2-Deoxy- $\beta$-D-erythro-pentofuranosyl)-6-[(4-pentylphenyl)ethynyl]furo[2,3- $d]$ pyrimidin-}

2(3H)-one [9b(iv)]. Treatment of 9a(iv) $(100 \mathrm{mg}, 0.20 \mathrm{mmol})$ with $\mathrm{NH}_{3} / \mathrm{MeOH}(20 \mathrm{~mL})$ by procedure B [5.5 h, chromatography $(\mathrm{MeOH} /$ EtOAc, 1:20 $\rightarrow$ 1:15)] gave 9b(iv) as yellow solid (61 mg, 70\%): UV max 354, $291 \mathrm{~nm}(\varepsilon 30500,31300), \min 321,253 \mathrm{~nm}(\varepsilon 13800,12900) ;{ }^{1} \mathrm{H}$ NMR (DMSO- $\left.d_{6}\right) \delta 8.89(\mathrm{~s}, 1 \mathrm{H}), 7.53(\mathrm{~d}, J=8.3 \mathrm{~Hz}, 2 \mathrm{H}), 7.29(\mathrm{~d}, J=8.3 \mathrm{~Hz}, 2 \mathrm{H}), 7.18(\mathrm{~s}, 1 \mathrm{H})$, $6.14(\mathrm{t}, J=6.0 \mathrm{~Hz}, 1 \mathrm{H}), 5.28(\mathrm{~d}, J=4.5 \mathrm{~Hz}, 1 \mathrm{H}), 5.13(\mathrm{t}, J=5.3 \mathrm{~Hz}, 1 \mathrm{H}), 4.25-4.22(\mathrm{~m}, 1 \mathrm{H})$, $3.94(\mathrm{dd}, J=3.8,7.8 \mathrm{~Hz}, 1 \mathrm{H}), 3.71-3.67(\mathrm{~m}, 1 \mathrm{H}), 3.65-3.60(\mathrm{~m}, 1 \mathrm{H}), 2.62(\mathrm{t}, J=7.5 \mathrm{~Hz}, 2 \mathrm{H})$, $2.42(\mathrm{ddd}, J=4.4,6.1,13.6 \mathrm{~Hz}, 1 \mathrm{H}), 2.09$ (dt, $J=6.5,13.0 \mathrm{~Hz}, 1 \mathrm{H}), 1.58$ (pent, $J=7.4 \mathrm{~Hz}, 2 \mathrm{H})$, $1.33-1.23(\mathrm{~m}, 4 \mathrm{H}), 0.86(\mathrm{t}, J=7.3 \mathrm{~Hz}, 3 \mathrm{H}) ;{ }^{13} \mathrm{C}$ NMR $\left(\mathrm{DMSO}-d_{6}\right) \delta 170.4,153.7,145.0,139.5$, $136.2,131.5,128.9,117.4,110.2,105.2,96.8,88.3,87.9,78.0,69.6,60.7,41.2,35.0,30.8,30.2$, 21.9, 13.8; HRMS (FAB) $m / z 445.1749\left(\mathrm{M}+\mathrm{Na}^{+}\left[\mathrm{C}_{24} \mathrm{H}_{26} \mathrm{~N}_{2} \mathrm{O}_{5} \mathrm{Na}\right]=445.1740\right)$. Anal. $\left(\mathrm{C}_{24} \mathrm{H}_{26} \mathrm{~N}_{2} \mathrm{O}_{5}\right) \mathrm{C}, \mathrm{H}, \mathrm{N}$.

\section{3-(3,5-Di- $O$-acetyl-2-deoxy- $\beta$-D-erythro-pentofuranosyl)-6-(2-phenylethyl)furo[2,3-}

d]pyrimidin-2(3H)-one [10a(i)]. Treatment of 9a(i) (136 mg, $0.312 \mathrm{mmol}), 10 \% \mathrm{Pd}-\mathrm{C}(14 \mathrm{mg})$, and $\mathrm{EtOH}(35 \mathrm{~mL})$ by procedure $\mathrm{C}$ [3 h, chromatography (EtOAc/hexanes, 3:2)] gave 10a(i) as a white solid (94 mg, 68\%): UV $\max 332,245 \mathrm{~nm}, \min 272,238 \mathrm{~nm} ;{ }^{1} \mathrm{H}$ NMR $\delta 8.15$ (s, 1H), $7.50-7.28(\mathrm{~m}, 2 \mathrm{H}), 7.23-7.18(\mathrm{~m}, 3 \mathrm{H}), 6.33(\mathrm{dd}, J=5.5,7.5 \mathrm{~Hz}, 1 \mathrm{H}), 6.06(\mathrm{~s}, 1 \mathrm{H}), 5.23(\mathrm{~d}, J=$ $6.0 \mathrm{~Hz}, 1 \mathrm{H}), 4.43-4.38(\mathrm{~m}, 3 \mathrm{H}), 3.05-2.94(\mathrm{~m}, 5 \mathrm{H}), 2.14-2.06(\mathrm{~m}, 1 \mathrm{H}), 2.13$ (s, 3H), 2.04 (s, $3 \mathrm{H}) ;{ }^{13} \mathrm{C}$ NMR $\delta 172.2,170.7,170.5,159.2,154.7,140.1,134.0,128.8,128.5,126.7,107.9$, 99.7, 88.6, 83.4, 74.3, 63.9, 39.5, 33.1, 30.3, 21.1, 21.0; HRMS (FAB) $m / z 463.1483\left(\mathrm{M}+\mathrm{Na}^{+}\right.$ $\left.\left[\mathrm{C}_{23} \mathrm{H}_{24} \mathrm{~N}_{2} \mathrm{O}_{7} \mathrm{Na}\right]=463.1481\right)$. 
3-(2-Deoxy- $\beta$-D-erythro-pentofuranosyl)-6-(2-phenylethyl)furo[2,3- $d]$ pyrimidin-2(3H)-one [10b(i)]. Treatment of 10a(i) $(86 \mathrm{mg}, 0.2 \mathrm{mmol})$ with $\mathrm{NH}_{3} / \mathrm{MeOH}(15 \mathrm{~mL})$ by procedure $\mathrm{B}[4.5$ h, chromatography $(\mathrm{MeOH} / \mathrm{EtOAc}, 1: 20 \rightarrow 1: 10)$ ] gave $\mathbf{1 0 b}(\mathbf{i})$ as a white solid $(60 \mathrm{mg}, 85 \%)$ : UV $\max 331,245 \mathrm{~nm}(\varepsilon 6700,13100), \min 272,239 \mathrm{~nm}(\varepsilon 600,12500) ;{ }^{1} \mathrm{H}$ NMR (DMSO- $\left.d_{6}\right) \delta$ $8.66(\mathrm{~s}, 1 \mathrm{H}), 7.29-7.24(\mathrm{~m}, 4 \mathrm{H}), 7.20-7.17(\mathrm{~m}, 1 \mathrm{H}), 6.39(\mathrm{~s}, 1 \mathrm{H}), 6.15(\mathrm{t}, J=6.3 \mathrm{~Hz}, 1 \mathrm{H}), 5.28$ $(\mathrm{d}, J=4.0 \mathrm{~Hz}, 1 \mathrm{H}), 5.12(\mathrm{t}, J=5.3 \mathrm{~Hz}, 1 \mathrm{H}), 4.23-4.20(\mathrm{~m}, 1 \mathrm{H}), 3.89(\mathrm{dd}, J=3.5,7.8 \mathrm{~Hz}, 1 \mathrm{H})$, $3.67(\mathrm{dt}, J=4.5,11.5 \mathrm{~Hz}, 1 \mathrm{H}), 3.60(\mathrm{dt}, J=4.5,12.2 \mathrm{~Hz}, 1 \mathrm{H}), 3.00-2.93(\mathrm{~m}, 4 \mathrm{H}), 2.37(\mathrm{ddd}, J=$ 4.3, 6.0, 13.5 Hz, 1H), $2.04(\mathrm{dt}, J=6.4,13.0 \mathrm{~Hz}, 1 \mathrm{H}) ;{ }^{13} \mathrm{C}$ NMR (DMSO- $\left.d_{6}\right) \delta 171.2,157.4$, $153.8,140.3,137.0,128.4,128.3,126.2,106.2,100.3,88.1,87.4,69.7,60.8,41.2,32.2,29.1$; HRMS $(F A B) \mathrm{m} / \mathrm{z} 379.1269\left(\mathrm{M}+\mathrm{Na}^{+}\left[\mathrm{C}_{19} \mathrm{H}_{20} \mathrm{~N}_{2} \mathrm{O}_{5} \mathrm{Na}\right]=379.1270\right)$. Anal. $\left(\mathrm{C}_{19} \mathrm{H}_{20} \mathrm{~N}_{2} \mathrm{O}_{5}\right) \mathrm{C}, \mathrm{H}$, N.

\section{3-(3,5-Di-O-acetyl-2-deoxy- $\beta$-D-erythro-pentofuranosyl)-6-[2-(4-}

methylphenyl)ethyl]furo[2,3-d]pyrimidin-2(3H)-one [10a(ii)]. Treatment of 9a(ii) (93 mg, $0.21 \mathrm{mmol}), 10 \% \mathrm{Pd}-\mathrm{C}(14 \mathrm{mg})$, and $\mathrm{EtOH}(28 \mathrm{~mL})$ by procedure $\mathrm{C}[4 \mathrm{~h}$, chromatography (EtOAc/hexanes, 3:2)] gave 10a(ii) as a white solid (64 mg, 67\%): UV max 321, 245, $225 \mathrm{~nm}$, $\min 274,238 \mathrm{~nm} ;{ }^{1} \mathrm{H}$ NMR $\delta 8.16(\mathrm{~s}, 1 \mathrm{H}), 7.11-7.07(\mathrm{~m}, 4 \mathrm{H}), 6.33(\mathrm{dd}, J=5.5,7.5 \mathrm{~Hz}, 1 \mathrm{H})$, $6.07(\mathrm{~s}, 1 \mathrm{H}), 5.22(\mathrm{~d}, J=6.0 \mathrm{~Hz}, 1 \mathrm{H}), 4.43-4.38(\mathrm{~m}, 3 \mathrm{H}), 2.99-2.94(\mathrm{~m}, 5 \mathrm{H}), 2.32(\mathrm{~s}, 3 \mathrm{H}), 2.13$ (s, 3H), 2.11-2.06 (m, 1H), $2.04(\mathrm{~s}, 3 \mathrm{H}) ;{ }^{13} \mathrm{C}$ NMR $\delta 172.2,170.7,170.5,159.3,154.7,137.0$, $136.2,133.9,129.5,128.4,107.9,99.6,88.6,83.4,74.3,63.9,39.5,32.7,30.4,21.2,21.1,21.0$; HRMS (FAB) $m / z$ 477.1626 $\left(\mathrm{M}+\mathrm{Na}^{+}\left[\mathrm{C}_{24} \mathrm{H}_{26} \mathrm{~N}_{2} \mathrm{O}_{7} \mathrm{Na}\right]=477.1638\right)$.

\section{3-(2-Deoxy- $\beta$-D-erythro-pentofuranosyl)-6-[2-(4-methylphenyl)ethyl]furo[2,3-}

d]pyrimidin-2(3H)-one [10b(ii)]. Treatment of $\mathbf{1 0 a}(\mathrm{ii})(74 \mathrm{mg}, 0.16 \mathrm{mmol})$ with $\mathrm{NH}_{3} / \mathrm{MeOH}$ $(12 \mathrm{~mL})$ by procedure $\mathrm{B}[3.5 \mathrm{~h}$, chromatography $(\mathrm{MeOH} / \mathrm{EtOAc}, 1: 20 \rightarrow 1: 10)]$ gave 10b(ii) as a 
white solid (47 mg, 79\%): UV max 331, $245 \mathrm{~nm}(\varepsilon 8100,16400)$, min 271, $238 \mathrm{~nm}(\varepsilon 800,15$ 600); ${ }^{1} \mathrm{H}$ NMR (DMSO- $\left.d_{6}\right) \delta 8.65(\mathrm{~s}, 1 \mathrm{H}), 7.13(\mathrm{~d}, J=8.0 \mathrm{~Hz}, 2 \mathrm{H}), 7.07(\mathrm{~d}, J=8.0 \mathrm{~Hz}, 2 \mathrm{H}), 6.37$ $(\mathrm{s}, 1 \mathrm{H}), 6.15(\mathrm{t}, J=6.5 \mathrm{~Hz}, 1 \mathrm{H}), 5.28(\mathrm{~d}, J=4.5 \mathrm{~Hz}, 1 \mathrm{H}), 5.11(\mathrm{t}, J=5.3 \mathrm{~Hz}, 1 \mathrm{H}), 4.23-4.21(\mathrm{~m}$, $1 \mathrm{H}), 3.89(\mathrm{dd}, J=4.0,7.5 \mathrm{~Hz}, 1 \mathrm{H}), 3.67-3.63(\mathrm{~m}, 1 \mathrm{H}), 3.62-3.58(\mathrm{~m}, 1 \mathrm{H}), 2.97-2.88(\mathrm{~m}, 4 \mathrm{H})$, $2.36(\mathrm{ddd}, J=4.0,6.3,13.5 \mathrm{~Hz}, 1 \mathrm{H}), 2.25(\mathrm{~s}, 3 \mathrm{H}), 2.03(\mathrm{dt}, J=6.0,13.3 \mathrm{~Hz}, 1 \mathrm{H}) ;{ }^{13} \mathrm{C}$ NMR $\left(\mathrm{DMSO}-d_{6}\right) \delta 171.1,157.5,153.8,137.2,137.0,135.1,128.9,128.2,106.2,100.3,88.1,87.4$, 69.7, 60.8, 41.2, 31.8, 29.1, 20.6; HRMS (FAB) $m / z$ 393.1445 $\left(\mathrm{M}+\mathrm{Na}^{+}\left[\mathrm{C}_{20} \mathrm{H}_{22} \mathrm{~N}_{2} \mathrm{O}_{5} \mathrm{Na}\right]=\right.$ 393.1426). Anal. $\left(\mathrm{C}_{20} \mathrm{H}_{22} \mathrm{~N}_{2} \mathrm{O}_{5}\right) \mathrm{C}, \mathrm{H}, \mathrm{N}$.

\section{3-(3,5-Di- $O$-acetyl-2-deoxy- $\beta$-D-erythro-pentofuranosyl)-6-[2-(4-}

propylphenyl)ethyl]furo[2,3-d]pyrimidin-2(3H)-one [10a(iii)]. Treatment of 9a(iii) (129 mg, $0.270 \mathrm{mmol}), 10 \% \mathrm{Pd}-\mathrm{C}(26 \mathrm{mg})$, and $\mathrm{EtOH}(25 \mathrm{~mL})$ by procedure $\mathrm{C}$ [ $5 \mathrm{~h}$, chromatography (EtOAc/hexanes, 3:2)] gave 10a(iii) as a white solid (80 mg, 63\%): UV max 331, 273, $246 \mathrm{~nm}$, $\min 291,238 \mathrm{~nm} ;{ }^{1} \mathrm{H}$ NMR $\delta 8.16(\mathrm{~s}, 1 \mathrm{H}), 7.10(\mathrm{bs}, 4 \mathrm{H}), 6.33$ (dd, J = 5.5, $\left.7.5 \mathrm{~Hz}, 1 \mathrm{H}\right), 6.08$ (s, $1 \mathrm{H}), 5.22(\mathrm{~d}, J=6.0 \mathrm{~Hz}, 1 \mathrm{H}), 4.42-4.38(\mathrm{~m}, 3 \mathrm{H}), 3.00-2.94(\mathrm{~m}, 5 \mathrm{H}), 2.60(\mathrm{t}, J=7.8 \mathrm{~Hz}, 2 \mathrm{H})$, 2.14-2.06 (m, 1H), $2.13(\mathrm{~s}, 3 \mathrm{H}), 2.05(\mathrm{~s}, 3 \mathrm{H}), 1.62$ (sext, $J=7.5 \mathrm{~Hz}, 2 \mathrm{H}), 0.94(\mathrm{t}, J=7.3 \mathrm{~Hz}$, $3 \mathrm{H}) ;{ }^{13} \mathrm{C}$ NMR $\delta 172.2,170.7,170.5,159.4,154.8,141.1,137.3,133.9,128.9,128.4,108.0$, 99.6, 88.7, 83.4, 74.3, 63.9, 39.5, 37.8, 32.7, 30.4, 24.8, 21.1, 21.0, 14.1; HRMS (FAB) $\mathrm{m} / z$ $505.1948\left(\mathrm{M}+\mathrm{Na}^{+}\left[\mathrm{C}_{26} \mathrm{H}_{30} \mathrm{~N}_{2} \mathrm{O}_{7} \mathrm{Na}\right]=505.1951\right)$.

\section{3-(2-Deoxy- $\beta$-D-erythro-pentofuranosyl)-6-[2-(4-propylphenyl)ethyl]furo[2,3- $d]$ pyrimidin-} 2(3H)-one [10b(iii)]. Treatment of 10a(iii) $(74 \mathrm{mg}, 0.15 \mathrm{mmol})$ with $\mathrm{NH}_{3} / \mathrm{MeOH}(10 \mathrm{~mL})$ by procedure B [8.5 h, chromatography $(\mathrm{MeOH} / \mathrm{EtOAc}, 1: 20)]$ gave $\mathbf{1 0 b}(\mathbf{i i i})$ as a white solid (43 mg, 73\%): UV max 331, 245, $225 \mathrm{~nm}(\varepsilon 8900,18400), \min 273,239 \mathrm{~nm}(\varepsilon 1200,17600) ;{ }^{1} \mathrm{H}$ NMR (DMSO- $\left.d_{6}\right) \delta 8.66(\mathrm{~s}, 1 \mathrm{H}), 7.15(\mathrm{~d}, J=8.3 \mathrm{~Hz}, 2 \mathrm{H}), 7.09(\mathrm{~d}, J=8.3 \mathrm{~Hz}, 2 \mathrm{H}), 6.40(\mathrm{~s}, 1 \mathrm{H})$, 
$6.16(\mathrm{t}, J=6.5 \mathrm{~Hz}, 1 \mathrm{H}), 5.28(\mathrm{~d}, J=4.5 \mathrm{~Hz}, 1 \mathrm{H}), 5.11(\mathrm{t}, J=5.5 \mathrm{~Hz}, 1 \mathrm{H}), 4.23-4.21(\mathrm{~m}, 1 \mathrm{H})$, $3.90(\mathrm{dd}, J=4.0,7.5 \mathrm{~Hz}, 1 \mathrm{H}), 3.68-3.64(\mathrm{~m}, 1 \mathrm{H}), 3.62-3.58(\mathrm{~m}, 1 \mathrm{H}), 2.98-2.96(\mathrm{~m}, 2 \mathrm{H})$, 2.92-2.89 (m, 2H), 2.54-2.48 (m, 2H, DMSO overlap), 2.37 (ddd, $J=4.1,6.1,13.6 \mathrm{~Hz}, 1 \mathrm{H})$, $2.04(\mathrm{dt}, J=6.4,13.0 \mathrm{~Hz}, 1 \mathrm{H}), 1.55(\mathrm{sext}, J=7.5 \mathrm{~Hz}, 2 \mathrm{H}), 0.87(\mathrm{t}, J=7.3 \mathrm{~Hz}, 3 \mathrm{H}) ;{ }^{13} \mathrm{C} \mathrm{NMR}$ $\left(\mathrm{DMSO}_{6}\right) \delta 171.2,157.5,153.8,139.9,137.5,137.0,128.3,128.2,106.3,100.2,88.1,87.4$, 69.7, 60.8, 41.2, 36.9, 31.8, 29.1, 24.1, 13.7; HRMS (FAB) $m / z 421.1734\left(\mathrm{M}+\mathrm{Na}^{+}\right.$ $\left.\left[\mathrm{C}_{22} \mathrm{H}_{26} \mathrm{~N}_{2} \mathrm{O}_{5} \mathrm{Na}\right]=421.1739\right)$. Anal. $\left(\mathrm{C}_{22} \mathrm{H}_{26} \mathrm{~N}_{2} \mathrm{O}_{5}\right) \mathrm{C}, \mathrm{H}, \mathrm{N}$.

\section{3-(3,5-Di-O-acetyl-2-deoxy- $\beta$-D-erythro-pentofuranosyl)-6-[2-(4-}

pentylphenyl)ethyl]furo[2,3- $d]$ pyrimidin-2(3H)-one [10a(iv)]. Treatment of 9a(iv) (53 mg, $0.11 \mathrm{mmol}), 10 \% \mathrm{Pd}-\mathrm{C}(8 \mathrm{mg})$, and $\mathrm{EtOH}(20 \mathrm{~mL})$ by procedure $\mathrm{C}[11 \mathrm{~h}$, chromatography (EtOAc/hexanes, 3:2)] gave 10a(iv) as a white solid (42 mg, 75\%): UV max 331, 245, $225 \mathrm{~nm}$, $\min 273,239 \mathrm{~nm} ;{ }^{1} \mathrm{H}$ NMR $\delta 8.15(\mathrm{~s}, 1 \mathrm{H}), 7.12-7.09(\mathrm{~m}, 4 \mathrm{H}), 6.33(\mathrm{dd}, J=5.5,7.5 \mathrm{~Hz}, 1 \mathrm{H})$, $6.08(\mathrm{~s}, 1 \mathrm{H}), 5.23(\mathrm{~d}, J=6.0 \mathrm{~Hz}, 1 \mathrm{H}), 4.42-4.38(\mathrm{~m}, 3 \mathrm{H}), 2.99-2.93(\mathrm{~m}, 5 \mathrm{H}), 2.60(\mathrm{t}, J=7.8 \mathrm{~Hz}$, 2H), $2.13(\mathrm{~s}, 3 \mathrm{H}), 2.12-2.06(\mathrm{~m}, 1 \mathrm{H}), 2.04(\mathrm{~s}, 3 \mathrm{H}), 1.59$ (pent, $J=7.4 \mathrm{~Hz}, 2 \mathrm{H}), 1.34-1.23$ (m, 4H), $0.89(\mathrm{t}, J=7.0 \mathrm{~Hz}, 3 \mathrm{H}) ;{ }^{13} \mathrm{C}$ NMR $\delta 172.2,170.7,170.5,159.4,154.7,141.3,137.3,133.9$, $128.8,128.3,107.9,99.6,88.6,83.4,74.3,63.9,39.5,35.7,32.7,31.7,31.4,30.4,22.7,21.1$, 21.0, 14.3; HRMS (FAB) $m / z$ 533.2269 ( $\left.\mathrm{M}+\mathrm{Na}^{+}\left[\mathrm{C}_{28} \mathrm{H}_{34} \mathrm{~N}_{2} \mathrm{O}_{7} \mathrm{Na}\right]=533.2264\right)$.

\section{3-(2-Deoxy- $\beta$-D-erythro-pentofuranosyl)-6-[2-(4-pentylphenyl)ethyl]furo[2,3- $d]$ pyrimidin-}

2(3H)-one [10b(iv)]. Treatment of 10a(iv) (39 mg, $0.076 \mathrm{mmol})$ with $\mathrm{NH}_{3} / \mathrm{MeOH}(10 \mathrm{~mL})$ by procedure $\mathrm{B}$ [3 h, chromatography (MeOH/EtOAc, 1:20)] gave 10b(iv) as a white solid (24 mg,

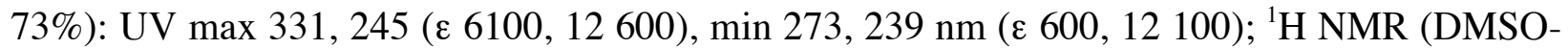
$\left.d_{6}\right) \delta 8.65(\mathrm{~s}, 1 \mathrm{H}), 7.14(\mathrm{~d}, J=8.0 \mathrm{~Hz}, 2 \mathrm{H}), 7.08(\mathrm{~d}, J=8.0 \mathrm{~Hz}, 2 \mathrm{H}), 6.39(\mathrm{~s}, 1 \mathrm{H}), 6.15(\mathrm{t}, J=6.3$ $\mathrm{Hz}, 1 \mathrm{H}), 5.29(\mathrm{~d}, J=4.5 \mathrm{~Hz}, 1 \mathrm{H}), 5.11(\mathrm{t}, J=5.3 \mathrm{~Hz}, 1 \mathrm{H}), 4.23-4.20(\mathrm{~m}, 1 \mathrm{H}), 3.89(\mathrm{dd}, J=3.8$, 
$7.8 \mathrm{~Hz}, 1 \mathrm{H}), 3.67-3.63(\mathrm{~m}, 1 \mathrm{H}), 3.61-3.57(\mathrm{~m}, 1 \mathrm{H}), 2.97-2.94(\mathrm{~m}, 2 \mathrm{H}), 2.91-2.88(\mathrm{~m}, 2 \mathrm{H})$, 2.53-2.49 (m, 2H), $2.36(\mathrm{ddd}, J=4.1,6.1,13.1 \mathrm{~Hz}, 1 \mathrm{H}), 2.03(\mathrm{dt}, J=6.5,13.0 \mathrm{~Hz}, 1 \mathrm{H}), 1.52$ (pent, $J=7.5 \mathrm{~Hz}, 2 \mathrm{H}), 1.30-1.20(\mathrm{~m}, 4 \mathrm{H}), 0.84(\mathrm{t}, J=7.0 \mathrm{~Hz}, 3 \mathrm{H}) ;{ }^{13} \mathrm{C}$ NMR (DMSO- $\left.d_{6}\right) \delta$ $171.2,157.6,153.8,140.1,137.5,137.0,128.3,128.2,106.3,100.3,88.1,87.4,69.7,60.8,41.2$, $34.8,31.8,30.9,30.7,29.1,22.0,14.0 ; \operatorname{HRMS}(\mathrm{FAB}) m / z 449.2048\left(\mathrm{M}+\mathrm{Na}^{+}\left[\mathrm{C}_{24} \mathrm{H}_{30} \mathrm{~N}_{2} \mathrm{O}_{5} \mathrm{Na}\right]=\right.$ 449.2052). Anal. $\left(\mathrm{C}_{24} \mathrm{H}_{30} \mathrm{~N}_{2} \mathrm{O}_{5}\right) \mathrm{C}, \mathrm{H}, \mathrm{N}$.

General Procedures for Synthesis of 14 and 15. We first employed a procedure reported for the synthesis of 2-bromo-5-methylpyridine, ${ }^{3}$ which involves lithiation of 3 -alkylpyridines ${ }^{4} \mathbf{1 2}$ (12 mmol) at C6 followed by C6 bromination. ${ }^{3}$ The resulting 5-alkyl-2-bromopyridine intermediates were subjected to Sonogashira coupling with TMS-acetylene followed by desilylation with TBAF to give 13.

(i) TMS-acetylene $(0.60 \mathrm{~mL}, 420 \mathrm{mg}, 4.25 \mathrm{mmol})$, and then $\left(\mathrm{Ph}_{3} \mathrm{P}\right)_{4} \mathrm{Pd}(190 \mathrm{mg}, 0.16 \mathrm{mmol})$ and $\mathrm{CuI}(22 \mathrm{mg}, 0.26 \mathrm{mmol})$ were added to a deoxygenated solution of 2-bromo-5-butylpyridine (700 mg, $3.27 \mathrm{mmol}$ ) in $\mathrm{CH}_{2} \mathrm{Cl}_{2} / \mathrm{Et}_{3} \mathrm{~N}\left(3: 2,10 \mathrm{~mL}\right.$ ), and the mixture was stirred at $50{ }^{\circ} \mathrm{C}$ for $2 \mathrm{~h}$. Volatiles were evaporated, and the residue was chromatographed (hexanes $\rightarrow$ EtOAc/hexanes, 1:10) to give an oil (650 mg), which was dissolved in THF (3 mL). (ii) TBAF/THF (1M, $3 \mathrm{~mL})$ was added to the solution, which was stirred for $30 \mathrm{~min}$. Volatiles were evaporated, and the residue was chromatographed (hexanes $\rightarrow$ EtOAc/hexanes, 1:10) to give 13a as an unstable oil [300 mg, 57\% (2 steps)]. (iii) $\mathrm{CH}_{2} \mathrm{Cl}_{2}(6 \mathrm{~mL})$ and $\mathrm{Et}_{3} \mathrm{~N}(5 \mathrm{~mL})$ were added to 1-[2-deoxy-3,5-di$O$-(p-toluoyl)- $\beta$-D-erythro-pentofuranosyl]-5-iodouracil $(900 \mathrm{mg}, 1.59 \mathrm{mmol})$ in a 30 -mL flask equipped with a Teflon valve, and $\mathrm{N}_{2}$ was bubbled through the stirred solution for 10 min. Addition of 13a $(254 \mathrm{mg}, 1.60 \mathrm{mmol})$ was followed by $\left(\mathrm{Ph}_{3} \mathrm{P}\right)_{4} \mathrm{Pd}(72 \mathrm{mg}, 0.06 \mathrm{mmol})$ and $\mathrm{CuI}$ (19 mg, $0.08 \mathrm{mmol}$ ), and stirring was continued for $1.5 \mathrm{~h}$ at $50{ }^{\circ} \mathrm{C}$. Volatiles were evaporated, 
and the residue was chromatographed (EtOAc/hexanes, 1:2 $\rightarrow$ EtOAc) to give 14a (745 mg, 97\%). (iv) This material (240 mg, $0.39 \mathrm{mmol})$ was stirred with $\mathrm{KOH}(65 \mathrm{mg}, 1.16 \mathrm{mmol})$ in $\mathrm{MeOH}(6.5 \mathrm{~mL})$ for $1 \mathrm{~h}$ at ambient temperature. The solution was neutralized $\left(37 \% \mathrm{HCl} / \mathrm{H}_{2} \mathrm{O}\right)$, and volatiles were evaporated. Chromatography (EtOAc $\rightarrow$ EtOAc/MeOH, 10:1) gave 5-[(5butylpyrid-2-yl)ethynyl]-1-(2-deoxy- $\beta$-D-erythro-pentofuranosyl)uracil (116 mg, 78\%). (v) A solution of this material $(230 \mathrm{mg}, 0.60 \mathrm{mmol})$ and $\mathrm{CuI}(113 \mathrm{mg}, 0.60 \mathrm{mmol})$ in DMF (3 mL) and $\mathrm{Et}_{3} \mathrm{~N}(3 \mathrm{~mL})$ was stirred for $3 \mathrm{~h}$ at $80{ }^{\circ} \mathrm{C}$. Volatiles were evaporated, and the residue was chromatographed (first with: EtOAc $\rightarrow$ EtOAc/MeOH, 10:1; and then with: $\mathrm{CH}_{2} \mathrm{Cl}_{2} \rightarrow$ $\left.\mathrm{CH}_{2} \mathrm{Cl}_{2} / \mathrm{MeOH}, 10: 1\right)$ to give $\mathbf{1 5 a}(220 \mathrm{mg}, 96 \%)$, which was recrystallized (MeOH).

\section{5-[2-(5-Butylpyrid-2-yl)ethynyl]-1-[2-deoxy-3,5-di-O-(p-toluoyl)- $\beta$-D-erythro-}

pentofuranosyl]uracil (14a). ${ }^{1} \mathrm{H}$ NMR $\delta 0.93(\mathrm{t}, J=7.3 \mathrm{~Hz}, 3 \mathrm{H}), 1.31-1.40(\mathrm{~m}, 2 \mathrm{H}), 1.54-1.64$ $(\mathrm{m}, 2 \mathrm{H}), 2.24,2.41(2 \times \mathrm{s}, 2 \times 3 \mathrm{H}), 2.24-2.40(\mathrm{~m}, 1 \mathrm{H}), 2.59(\mathrm{t}, J=7.8 \mathrm{~Hz}, 2 \mathrm{H}), 2.77(\mathrm{dd}, J=5.4$, $14.2 \mathrm{~Hz}, 1 \mathrm{H}), 4.57-4.59(\mathrm{~m}, 1 \mathrm{H}), 4.65(\mathrm{dd}, J=3.4,12.2 \mathrm{~Hz}, 1 \mathrm{H}), 4.79(\mathrm{dd}, J=3.9,12.2 \mathrm{~Hz}$, $1 \mathrm{H}), 5.59(\mathrm{~d}, J=6.3 \mathrm{~Hz}, 1 \mathrm{H}), 6.40(\mathrm{dd}, J=5.9,8.8 \mathrm{~Hz}, 1 \mathrm{H}), 7.14-7.28(\mathrm{~m}, 5 \mathrm{H}), 7.42(\mathrm{dd}, J=$ 2.0, 7.8 Hz, 1H), 7.91-7.94 (m, 4H), $8.08(\mathrm{~s}, 1 \mathrm{H}), 8.42$ (d, J = 2.0 Hz, 1H), 9.80 (br s, $1 \mathrm{H}) ;{ }^{13} \mathrm{C}$ NMR $\delta 13.6,21.3,21.5,22.0,32.3,32.7,37.9,64.0,74.7,80.0,82.9,85.6,92.3,99.9,126.1$, $126.2,126.6,129.0,129.1,129.4,129.5,135.6,137.4,139.8,142.7,143.8,144.1,149.4,149.7$, 161.1, 165.6, 165.9; FAB-MS m/z 622 ([M + H+ $], 10 \%), 270(100 \%) ;$ HRMS m/z $622.2550(\mathrm{M}+$ $\left.\mathrm{H}^{+}\left[\mathrm{C}_{36} \mathrm{H}_{36} \mathrm{~N}_{3} \mathrm{O}_{7}\right]=622.2553\right)$.

6-(5-Butylpyrid-2-yl)-3-(2-deoxy- $\beta$-D-erythro-pentofuranosyl)furo[2,3- $d]$ pyrimidin-2(3H)-

one (15a). ${ }^{1} \mathrm{H}$ NMR $\left(\mathrm{CD}_{3} \mathrm{OD}\right) \delta 0.96(\mathrm{t}, J=7.3 \mathrm{~Hz}, 3 \mathrm{H}), 1.35-1.43(\mathrm{~m}, 2 \mathrm{H}), 1.60-1.66(\mathrm{~m}, 2 \mathrm{H})$, $2.24(\mathrm{td}, J=6.1,13.7 \mathrm{~Hz}, 1 \mathrm{H}), 2.63(\mathrm{ddd}, J=4.4,5.9,12.2 \mathrm{~Hz}, 1 \mathrm{H}), 2.67(\mathrm{t}, J=7.8 \mathrm{~Hz}, 2 \mathrm{H})$, 3.81, $3.92(2 \times \mathrm{dd}, J=3.4,12.2 \mathrm{~Hz}, 2 \times 1 \mathrm{H}), 4.07-4.09(\mathrm{~m}, 1 \mathrm{H}), 4.39-4.23(\mathrm{~m}, 1 \mathrm{H}), 7.24,7.735$, 
7.738, 8.41, $9.05(5 \times \mathrm{s}, 5 \times 1 \mathrm{H}) ;{ }^{13} \mathrm{C} \mathrm{NMR}\left(\mathrm{CD}_{3} \mathrm{OD}\right) \delta 14.3,23.5,33.6,34.5,43.0,62.4,71.4$, $89.9,90.2,102.9,109.3,121.3,138.8,140.8,140.9,146.1,151.2,155.6,156.9,172.9$; FAB-MS $m / z 408\left(\left[\mathrm{M}+\mathrm{Na}^{+}\right], 45 \%\right), 270(100 \%) ;$ HRMS m/z $408.1520\left(\mathrm{M}+\mathrm{Na}^{+}\left[\mathrm{C}_{20} \mathrm{H}_{23} \mathrm{~N}_{3} \mathrm{O}_{5} \mathrm{Na}\right]=\right.$ 408.1535).

\section{1-[2-Deoxy-3,5-di-O-(p-toluoyl)- $\beta$-D-erythro-pentofuranosyl]-5-[2-(5-pentylpyrid-2-}

yl)ethynyl]uracil (14b). ${ }^{1} \mathrm{H}$ NMR $\delta 0.90(\mathrm{t}, J=7.3 \mathrm{~Hz}, 3 \mathrm{H}), 1.29-1.38(\mathrm{~m}, 4 \mathrm{H}), 1.57-1.65(\mathrm{~m}$, $2 \mathrm{H}), 2.25,2.43(2 \times \mathrm{s}, 2 \times 3 \mathrm{H}), 2.29-2.35(\mathrm{~m}, 1 \mathrm{H}), 2.60(\mathrm{t}, J=7.8 \mathrm{~Hz}, 2 \mathrm{H}), 2.77(\mathrm{dd}, J=5.4$, $14.2 \mathrm{~Hz}, 1 \mathrm{H}), 4.57-4.59(\mathrm{~m}, 1 \mathrm{H}), 4.65(\mathrm{dd}, J=3.4,12.2 \mathrm{~Hz}, 1 \mathrm{H}), 4.80(\mathrm{dd}, J=3.9,12.2 \mathrm{~Hz}$, $1 \mathrm{H}), 5.60(\mathrm{~d}, J=6.3 \mathrm{~Hz}, 1 \mathrm{H}), 6.39(\mathrm{dd}, J=5.9,8.8 \mathrm{~Hz}, 1 \mathrm{H}), 7.15-7.30(\mathrm{~m}, 5 \mathrm{H}), 7.43(\mathrm{dd}, J=$ 2.0, $7.8 \mathrm{~Hz}, 1 \mathrm{H}), 7.91-7.94(\mathrm{~m}, 4 \mathrm{H}), 8.06(\mathrm{~s}, 1 \mathrm{H}), 8.42(\mathrm{~d}, J=2.0 \mathrm{~Hz}, 1 \mathrm{H}), 8.15$ (br s, $1 \mathrm{H}) ;{ }^{13} \mathrm{C}$ NMR $\delta 13.9,21.5,21.7,22.3,30.5,31.2,32.8,38.4,64.0,74.8,79.5,83.2,85.8,92.8,100.2$, $126.2,126.3,126.8,129.2,129.3,129.5,129.7,135.7,137.7,139.9,142.4,144.1,144.4,149.2$ 150.0, 161.0, 165.8, 166.1; FAB-MS m/z $636\left(\left[\mathrm{M}+\mathrm{H}^{+}\right], 40 \%\right), 284(100 \%) ; \mathrm{HRMS} \mathrm{m} / \mathrm{z}$ 636.2708 $\left(\mathrm{M}+\mathrm{H}^{+}\left[\mathrm{C}_{37} \mathrm{H}_{38} \mathrm{~N}_{3} \mathrm{O}_{7}\right]=636.2709\right)$.

\section{3-(2-Deoxy- $\beta$-D-erythro-pentofuranosyl)-6-(5-pentylpyrid-2-yl)furo[2,3- $d]$ pyrimidin-}

2(3H)-one (15b). ${ }^{1} \mathrm{H}$ NMR (DMSO- $\left.d_{6}\right) \delta 0.85(\mathrm{t}, J=7.3 \mathrm{~Hz}, 3 \mathrm{H}), 1.24-1.36(\mathrm{~m}, 4 \mathrm{H}), 1.56-1.64$ $(\mathrm{m}, 2 \mathrm{H}), 2.12(\mathrm{td}, J=6.1,13.7 \mathrm{~Hz}, 1 \mathrm{H}), 2.42-2.46(\mathrm{~m}, 1 \mathrm{H}), 2.41-2.44(\mathrm{~m}, 1 \mathrm{H}), 2.63(\mathrm{t}, J=7.8$ $\mathrm{Hz}, 2 \mathrm{H}), 3.63-3.76(\mathrm{~m}, 2 \mathrm{H}), 3.95-4.00(\mathrm{~m}, 1 \mathrm{H}), 4.25-4.29(\mathrm{~m}, 1 \mathrm{H}), 5.19(\mathrm{t}, J=5.4 \mathrm{~Hz}, 1 \mathrm{H}), 5.34$ $(\mathrm{d}, J=4.4 \mathrm{~Hz}, 1 \mathrm{H}), 6.19(\mathrm{t}, J=6.1 \mathrm{~Hz}, 1 \mathrm{H}), 7.30,8.52,8.92(3 \times \mathrm{s}, 3 \times 1 \mathrm{H}), 7.74-7.78(\mathrm{~m}, 2 \mathrm{H})$; ${ }^{13} \mathrm{C}$ NMR (DMSO- $\left.d_{6}\right) \delta 13.9,21.9,30.2,30.8,32.0,41.2,60.8,69.7,87.8,88.3,101.7,106.4$, $119.4,136.9,138.3,139.3,144.6,150.2,153.3,153.8,171.1$; FAB-MS $m / z 422\left(\left[\mathrm{M}+\mathrm{Na}^{+}\right]\right.$, 45\%), $270(100 \%) ; \mathrm{HRMS} m / z 422.1700\left(\mathrm{M}+\mathrm{Na}^{+}\left[\mathrm{C}_{21} \mathrm{H}_{25} \mathrm{~N}_{3} \mathrm{O}_{5} \mathrm{Na}\right]=422.1692\right)$. 


\section{1-[2-Deoxy-3,5-di-O-(p-toluoyl)- $\beta$-D-erythro-pentofuranosyl]-5-[2-(5-hexylpyrid-2-}

yl)ethynyl]uracil (14c). ${ }^{1} \mathrm{H}$ NMR $\delta 0.88(\mathrm{t}, J=7.3 \mathrm{~Hz}, 3 \mathrm{H}), 1.26-1.38(\mathrm{~m}, 6 \mathrm{H}), 1.56-1.65(\mathrm{~m}$, 2H), 2.25, $2.43(2 \times \mathrm{s}, 2 \times 3 \mathrm{H}), 2.30-2.36(\mathrm{~m}, 1 \mathrm{H}), 2.59(\mathrm{t}, J=7.8 \mathrm{~Hz}, 2 \mathrm{H}), 2.77(\mathrm{dd}, J=5.4$, 14.2 Hz, 1H), 4.56-4.58 (m, 1H), 4.65, $4.80(2 \times \mathrm{dd}, J=3.4,12.2 \mathrm{~Hz}, 2 \times 1 \mathrm{H}), 5.60(\mathrm{~d}, J=6.3$ $\mathrm{Hz}, 1 \mathrm{H}), 6.39(\mathrm{dd}, J=5.9,8.8 \mathrm{~Hz}, 1 \mathrm{H}), 7.15-7.30(\mathrm{~m}, 5 \mathrm{H}), 7.43(\mathrm{dd}, J=2.0,7.8 \mathrm{~Hz}, 1 \mathrm{H})$, 7.91-7.94 (m, 4H), $8.06(\mathrm{~s}, 1 \mathrm{H}), 8.41(\mathrm{~d}, J=2.0 \mathrm{~Hz}, 1 \mathrm{H}), 8.99(\mathrm{br} \mathrm{s}, 1 \mathrm{H}) ;{ }^{13} \mathrm{C}$ NMR $\delta 14.0,21.5$, 21.6, 22.5, 28.7, 30.8, 31.5, 32.8, 38.3, 64.1, 74.8, 79.6, 83.1, 85.8, 92.7, 100.2, $126.2(2 \mathrm{C})$, $126.3,126.8,129.2,129.4,129.5,129.7,135.7,137.7,139.9,142.5,144.1,144.4,149.3,150.0$, 161.0, 165.8, 166.1; FAB-MS m/z 650 ([M + H+ $], 50 \%), 298(100 \%)$; HRMS m/z 650.2858 (M + $\left.\mathrm{H}^{+}\left[\mathrm{C}_{38} \mathrm{H}_{40} \mathrm{~N}_{3} \mathrm{O}_{7}\right]=650.2866\right)$.

\section{3-(2-Deoxy- $\beta$-D-erythro-pentofuranosyl)-6-(5-hexylpyrid-2-yl)furo[2,3- $d]$ pyrimidin-2(3H)-}

one (15c). ${ }^{1} \mathrm{H}$ NMR (DMSO- $\left.d_{6}\right) \delta 0.85(\mathrm{t}, J=7.3 \mathrm{~Hz}, 3 \mathrm{H}), 1.24-1.36(\mathrm{~m}, 6 \mathrm{H}), 1.55-1.64(\mathrm{~m}$, 2H), $2.12(\mathrm{td}, J=6.1,13.7 \mathrm{~Hz}, 1 \mathrm{H}), 2.42-2.47(\mathrm{~m}, 1 \mathrm{H}), 2.41-2.44(\mathrm{~m}, 1 \mathrm{H}), 2.63(\mathrm{t}, J=7.8 \mathrm{~Hz}$, 2H), 3.63-3.75 (m, 2H), 3.95-3.98 (m, 1H), 4.25-4.29 (m, 1H), $5.19(\mathrm{t}, J=5.4 \mathrm{~Hz}, 1 \mathrm{H}), 5.34(\mathrm{~d}$, $J=4.4 \mathrm{~Hz}, 1 \mathrm{H}), 6.19(\mathrm{t}, J=6.1 \mathrm{~Hz}, 1 \mathrm{H}), 7.30,8.52,8.92(3 \times \mathrm{s}, 3 \times 1 \mathrm{H}), 7.74-7.78(\mathrm{~m}, 2 \mathrm{H}) ;{ }^{13} \mathrm{C}$ NMR $\left(\mathrm{DMSO}-d_{6}\right) \delta 14.0,22.1,28.3,30.5,31.1,32.0,41.2,60.8,69.7,87.8,88.3,101.7,106.4$, $119.4,136.9,138.3,139.3,144.6,150.2,153.3,153.8,171.1 ; \mathrm{FAB}-\mathrm{MS} m / z 436\left(\left[\mathrm{M}+\mathrm{Na}^{+}\right]\right.$, 25\%), $298(100 \%) ; \mathrm{HRMS} m / z 436.1848\left(\mathrm{M}+\mathrm{Na}^{+}\left[\mathrm{C}_{22} \mathrm{H}_{27} \mathrm{~N}_{3} \mathrm{O}_{5} \mathrm{Na}\right]=436.1848\right)$.

General Procedures for Synthesis of 20. (i) A solution of 5-bromo-2-iodopyridine (6) (1.6 g, $5.63 \mathrm{mmol})$ in $\mathrm{CH}_{2} \mathrm{Cl}_{2}(10 \mathrm{~mL})$ and $\mathrm{Et}_{3} \mathrm{~N}(8 \mathrm{~mL})$ was deoxygenated with a stream of nitrogen. Addition of 1-hexyne $(0.50 \mathrm{~g}, 0.70 \mathrm{~mL}, 6.1 \mathrm{mmol})$ was followed by $\left(\mathrm{Ph}_{3} \mathrm{P}\right)_{4} \mathrm{Pd}(320 \mathrm{mg}, 0.28$ mmol, 0.05 equiv) and $\mathrm{CuI}(80 \mathrm{mg}, 0.42 \mathrm{mmol}, 0.07$ equiv) and the mixture was stirred for $7 \mathrm{~h}$ at $60{ }^{\circ} \mathrm{C}$. Volatiles were evaporated, and the residue was chromatographed (EtOAc/hexanes, 1:6) to 
give crude 5-bromo-2-(hex-1-yn-1-yl)pyridine (17b) (1.34 g): ${ }^{1} \mathrm{H}$ NMR $\delta 0.94$ (m, 3H), $1.45-1.52(\mathrm{~m}, 2 \mathrm{H}), 1.58-1.64(\mathrm{~m}, 2 \mathrm{H}), 2.43(\mathrm{t}, J=7.8 \mathrm{~Hz}, 2 \mathrm{H}), 7.25(\mathrm{~d}, J=8.3 \mathrm{~Hz}, 1 \mathrm{H}), 7.74$ $(\mathrm{dd}, J=2.4,8.3 \mathrm{~Hz}, 1 \mathrm{H}), 8.59(\mathrm{~d}, J=2.4 \mathrm{~Hz}, 1 \mathrm{H}) ;{ }^{13} \mathrm{C} \mathrm{NMR} \delta$ 13.2, 18.6, 21.6, 29.8, 79.1, 92.0, $118.8,127.3,138.1,141.8,150.3$.

(ii) A solution of this material in $\mathrm{EtOH}(25 \mathrm{~mL})$ and $\mathrm{Et}_{3} \mathrm{~N}(0.5 \mathrm{~mL})$ was hydrogenated ${ }^{5}$ over $\mathrm{PtO}_{2}(80 \mathrm{mg})$ for $24 \mathrm{~h}$. Volatiles were evaporated to give 5-bromo-2-hexylpyridine (17d): ${ }^{1} \mathrm{H}$ NMR $\delta 0.86-0.92(\mathrm{~m}, 3 \mathrm{H}), 1.24-1.38(\mathrm{~m}, 6 \mathrm{H}), 1.66-1.74(\mathrm{~m}, 2 \mathrm{H}), 2.74(\mathrm{t}, J=7.8 \mathrm{~Hz}, 2 \mathrm{H}), 7.05$ $(\mathrm{d}, J=8.3 \mathrm{~Hz}, 1 \mathrm{H}), 7.07(\mathrm{dd}, J=2.4,8.3 \mathrm{~Hz}, 1 \mathrm{H}), 8.57(\mathrm{~d}, J=2.4 \mathrm{~Hz}, 1 \mathrm{H}) ;{ }^{13} \mathrm{C}$ NMR $\delta 13.8$ $22.3,28.8,29.5,31.4,37.5,117.5,123.7,138.4,149.9,160.8$; FAB-MS $m / z 242\left(\left[\mathrm{M}+\mathrm{H}^{+}\right]\right.$ $100 \%) ; \mathrm{HRMS} m / z, 242.0538\left(\mathrm{M}+\mathrm{H}^{+}\left[\mathrm{C}_{11} \mathrm{H}_{17} \mathrm{BrN}\right]=242.0539\right)$.

[The intermediate 2-(2-alkylpyrid-5-yl)ethynes $18 \mathbf{c}$ and 18d were prepared from the respective 2-alkyl-5-bromopyridines $\mathbf{1 7} \mathbf{c}$ and $\mathbf{1 7 d}$ (by the procedure described for the conversion of 5-alkyl2-bromopyridines $\rightarrow 13$ ) and used directly (18c and $18 d \rightarrow 19 \mathrm{c}$ and 19d). The pyridine-2,5diyne 18a was obtained from $17 \mathbf{a}$ by the same procedure.]

(iii) A 30-mL round-bottom flask equipped with a Teflon valve was charged with 1-[2-deoxy3,5-di-O-(p-toluoyl)- $\beta$-D-erythro-pentofuranosyl]-5-iodouracil (1.00 g, $1.76 \mathrm{mmol}), \mathrm{CH}_{2} \mathrm{Cl}_{2}$ (6 $\mathrm{mL}$ ), and $\mathrm{Et}_{3} \mathrm{~N}(5 \mathrm{~mL})$, and $\mathrm{N}_{2}$ was bubbled through the stirred solution for $10 \mathrm{~min}$. Alkyne 18a (300 mg, $1.75 \mathrm{mmol})$ was added followed by $\left(\mathrm{Ph}_{3} \mathrm{P}\right)_{4} \mathrm{Pd}(100 \mathrm{mg}, 0.09 \mathrm{mmol}, 0.05$ equiv) and $\mathrm{CuI}$ (27 mg, $0.14 \mathrm{mmol}, 0.08$ equiv). Stirring was continued for $7 \mathrm{~h}$ at $60^{\circ} \mathrm{C}$, and volatiles were evaporated. The residue was chromatographed (EtOAc/hexanes, 1:2 $\rightarrow$ EtOAc) to give 19a (0.85 $\mathrm{g}, 79 \%)$. (iv) This material was stirred for $1 \mathrm{~h}$ in a solution of $\mathrm{KOH}(150 \mathrm{mg}, 2.68 \mathrm{mmol})$ in $\mathrm{MeOH}(20 \mathrm{~mL})$. Neutralization $\left(37 \% \mathrm{HCl} / \mathrm{H}_{2} \mathrm{O}\right)$, evaporation of volatiles, and chromatography $(\mathrm{EtOAc} \rightarrow \mathrm{EtOAc} / \mathrm{MeOH}, 10: 1)$ gave a solid (510 mg, 92\%). (v) A solution of this material (510 
$\mathrm{mg}, 1.28 \mathrm{mmol})$ and $\mathrm{CuI}(240 \mathrm{mg}, 1.28 \mathrm{mmol})$ in $\mathrm{DMF}(3.5 \mathrm{~mL})$ and $\mathrm{Et}_{3} \mathrm{~N}(3.5 \mathrm{~mL})$ was stirred for $4 \mathrm{~h}$ at $80{ }^{\circ} \mathrm{C}$. Volatiles were evaporated, and the residue was chromatographed (first with: EtOAc $\rightarrow$ EtOAc/MeOH, 10:1; second with: $\mathrm{CH}_{2} \mathrm{Cl}_{2} \rightarrow \mathrm{CH}_{2} \mathrm{Cl}_{2} / \mathrm{MeOH}, 10: 1$ ) to give 20a (460 mg, 90\%), which was recrystallized $(\mathrm{MeOH})$.

\section{3-(2-Deoxy- $\beta$-D-erythro-pentofuranosyl)-6-[2-(pent-1-yn-1-yl)pyrid-5-yl]furo[2,3-}

d]pyrimidin-2(3H)-one (20a). ${ }^{1} \mathrm{H}$ NMR (DMSO- $\left.d_{6}\right) \delta 1.02(\mathrm{t}, J=7.3 \mathrm{~Hz}, 3 \mathrm{H}), 1.56-1.64(\mathrm{~m}$, 2H), $2.12(\mathrm{td}, J=6.1,13.2 \mathrm{~Hz}, 1 \mathrm{H}), 2.40-2.53(\mathrm{~m}, 4 \mathrm{H}), 3.62-3.75(\mathrm{~m}, 2 \mathrm{H}), 3.92-3.96(\mathrm{~m}, 1 \mathrm{H})$, $4.24-4.28(\mathrm{~m}, 1 \mathrm{H}), 5.23(\mathrm{t}, J=5.1 \mathrm{~Hz}, 1 \mathrm{H}), 5.33(\mathrm{~d}, J=4.4 \mathrm{~Hz}, 1 \mathrm{H}), 6.18(\mathrm{t}, J=6.1 \mathrm{~Hz}, 1 \mathrm{H})$, 7.49, $8.96(2 \times \mathrm{s}, 2 \times 1 \mathrm{H}), 7.57(\mathrm{~d}, J=8.3 \mathrm{~Hz}, 1 \mathrm{H}), 8.15(\mathrm{dd}, J=1.9,8.3 \mathrm{~Hz}, 1 \mathrm{H}), 9.00($ br s, $1 \mathrm{H}) ;{ }^{13} \mathrm{C}$ NMR $\left(\mathrm{DMSO}-d_{6}\right) \delta 13.4,20.5,21.4,41.3,60.6,69.4,80.9,87.8,88.2,92.5,102.0$, $106.4,122.3,126.9,131.9,139.1,142.7,145.9,150.6,153.7,171.0 ;$ FAB-MS m/z $418([\mathrm{M}+$ $\left.\left.\mathrm{Na}^{+}\right], 25 \%\right), 279(100 \%)$; HRMS $m / z 418.1378\left(\mathrm{M}+\mathrm{Na}^{+}\left[\mathrm{C}_{21} \mathrm{H}_{21} \mathrm{~N}_{3} \mathrm{O}_{5} \mathrm{Na}\right]=418.1379\right)$.

\section{3-(2-Deoxy- $\beta$-D-erythro-pentofuranosyl)-6-(2-pentylpyrid-5-yl)furo[2,3- $d]$ pyrimidin-}

2(3H)-one (20c). ${ }^{1} \mathrm{H}$ NMR (DMSO- $\left.d_{6}\right) \delta 0.87(\mathrm{t}, J=7.3 \mathrm{~Hz}, 3 \mathrm{H}), 1.25-1.37(\mathrm{~m}, 4 \mathrm{H}), 1.64-1.74$ $(\mathrm{m}, 2 \mathrm{H}), 2.12(\mathrm{td}, J=6.1,13.2 \mathrm{~Hz}, 1 \mathrm{H}), 2.40-2.46(\mathrm{~m}, 1 \mathrm{H}), 2.50-2.54(\mathrm{~m}, 1 \mathrm{H}), 2.77(\mathrm{t}, J=7.8$ $\mathrm{Hz}, 2 \mathrm{H}), 3.62-3.76(\mathrm{~m}, 2 \mathrm{H}), 3.92-3.96(\mathrm{~m}, 1 \mathrm{H}), 4.24-4.29(\mathrm{~m}, 1 \mathrm{H}), 5.18(\mathrm{t}, J=5.4 \mathrm{~Hz}, 1 \mathrm{H})$, $5.30(\mathrm{~d}, J=4.4 \mathrm{~Hz}, 1 \mathrm{H}), 6.19(\mathrm{t}, J=6.1 \mathrm{~Hz}, 1 \mathrm{H}), 7.36,8.91(2 \times \mathrm{s}, 2 \times 1 \mathrm{H}), 7.39(\mathrm{~d}, J=8.3 \mathrm{~Hz}$, $1 \mathrm{H}), 8.08(\mathrm{dd}, J=1.9,8.3 \mathrm{~Hz}, 1 \mathrm{H}), 8.95($ br s, $1 \mathrm{H}) ;{ }^{13} \mathrm{C}$ NMR $\left(\mathrm{DMSO}-d_{6}\right) \delta 13.9,21.9,28.6$, $30.9,37.3,41.3,60.6,69.4,87.7,88.2,100.3,106.5,122.1,122.8,132.1,138.5,145.1,151.4$, 153.7, 162.6, 171.0; FAB-MS m/z, $400\left(\left[\mathrm{M}+\mathrm{H}^{+}\right], 35 \%\right), 117(100 \%)$; HRM $m / z 400.1882(\mathrm{M}+$ $\left.\mathrm{H}^{+}\left[\mathrm{C}_{21} \mathrm{H}_{26} \mathrm{~N}_{3} \mathrm{O}_{5}\right]=400.1867\right)$.

3-(2-Deoxy- $\beta$-D-erythro-pentofuranosyl)-6-(2-hexylpyrid-5-yl)furo[2,3- $d]$ pyrimidin-2(3H)one (20d). ${ }^{1} \mathrm{H}$ NMR (DMSO- $\left.d_{6}\right) \delta 0.85(\mathrm{t}, J=7.3 \mathrm{~Hz}, 3 \mathrm{H}), 1.22-1.38(\mathrm{~m}, 6 \mathrm{H}), 1.52-1.72(\mathrm{~m}$, 
2H), $2.11(\mathrm{td}, J=6.1,13.2 \mathrm{~Hz}, 1 \mathrm{H}), 2.39-2.45(\mathrm{~m}, 1 \mathrm{H}), 2.48-2.53(\mathrm{~m}, 1 \mathrm{H}), 2.76(\mathrm{t}, J=7.8 \mathrm{~Hz}$, $2 \mathrm{H}), 3.61-3.75(\mathrm{~m}, 2 \mathrm{H}), 3.92-3.96(\mathrm{~m}, 1 \mathrm{H}), 4.23-4.28(\mathrm{~m}, 1 \mathrm{H}), 5.17(\mathrm{t}, J=5.4 \mathrm{~Hz}, 1 \mathrm{H}), 5.29(\mathrm{~d}$, $J=4.4 \mathrm{~Hz}, 1 \mathrm{H}), 6.18(\mathrm{t}, J=6.1 \mathrm{~Hz}, 1 \mathrm{H}), 7.36,8.90(2 \times \mathrm{s}, 2 \times 1 \mathrm{H}), 7.39(\mathrm{~d}, J=7.8 \mathrm{~Hz}, 1 \mathrm{H})$, $8.08(\mathrm{dd}, J=1.5,7.8 \mathrm{~Hz}, 1 \mathrm{H}), 8.96$ (br s, $1 \mathrm{H}) ;{ }^{13} \mathrm{C}$ NMR (DMSO- $d_{6}$ ) $\delta 13.9,22.0,28.3,28.9$, $31.1,37.3,41.3,60.6,69.5,87.7,88.2,100.3,106.5,122.1,122.8,132.1,138.5,145.1,151.5$, 153.7, 162.6, 171.0; FAB-MS $m / z 414\left(\left[\mathrm{M}+\mathrm{H}^{+}\right], 100 \%\right)$; HRMS $m / z 414.2020\left(\mathrm{M}+\mathrm{H}^{+}\right.$ $\left.\left[\mathrm{C}_{22} \mathrm{H}_{28} \mathrm{~N}_{3} \mathrm{O}_{5}\right]=414.2024\right)$.

General Procedures for Synthesis of 22 and 23. (i) A solution of 2-amino-5bromopyrimidine (21) $(1.6 \mathrm{~g}, 9.2 \mathrm{mmol})$ in DMSO $(12 \mathrm{~mL})$ and $\mathrm{Et}_{3} \mathrm{~N}(6 \mathrm{~mL})$ was deoxygenated with a stream of $\mathrm{N}_{2}$. Addition of 1-pentyne $(0.70 \mathrm{~g}, 1.0 \mathrm{~mL}, 10.25 \mathrm{mmol})$ was followed by $\left(\mathrm{Ph}_{3} \mathrm{P}\right)_{4} \mathrm{Pd}(530 \mathrm{mg}, 0.46 \mathrm{mmol})$ and $\mathrm{CuI}(140 \mathrm{mg}, 0.74 \mathrm{mmol})$. The mixture was stirred for $5 \mathrm{~h}$ at $55^{\circ} \mathrm{C}$, and volatiles were evaporated. The residue was suspended in hot $\mathrm{MeOH}$ and filtered. Recrystallization $(\mathrm{MeOH})$ gave 2-amino-5-(pent-1-yn-1-yl)pyrimidine (1.67 g, 79\%): ${ }^{1} \mathrm{H}$ NMR $\delta$ $1.04(\mathrm{t}, J=7.3 \mathrm{~Hz}, 3 \mathrm{H}), 1.58-1.66(\mathrm{~m}, 2 \mathrm{H}), 2.38(\mathrm{t}, J=6.8 \mathrm{~Hz}, 2 \mathrm{H}), 5.20($ br s, $2 \mathrm{H}), 8.32(\mathrm{~s}$, $2 \mathrm{H}) ;{ }^{13} \mathrm{C}$ NMR $\delta 13.5,21.4,22.1,74.6,93.5,109.9,160.4,160.9 ;$ FAB-MS $m / z .162\left(\left[\mathrm{M}+\mathrm{H}^{+}\right]\right.$, 100\%); HRMS $m / z 162.1025\left(\mathrm{M}+\mathrm{H}^{+}\left[\mathrm{C}_{9} \mathrm{H}_{12} \mathrm{~N}_{3}\right]=162.1031\right)$. (ii) A solution of this material (700 mg, $4.35 \mathrm{mmol})$ in $\mathrm{MeOH}(20 \mathrm{~mL})$ was hydrogenated at 60 psi over 10\% Pd-C (80 mg) for $24 \mathrm{~h}$. Volatiles were evaporated, and the residue was chromatographed $\left(\mathrm{CH}_{2} \mathrm{Cl}_{2} \rightarrow\right.$ EtOAc) to give 2-Amino-5-pentylpyrimidine (22a): ${ }^{1} \mathrm{H}$ NMR $\delta 0.84(\mathrm{t}, J=6.8 \mathrm{~Hz}, 3 \mathrm{H}), 1.22-1.34(\mathrm{~m}$, $4 \mathrm{H}), 1.46-1.55(\mathrm{~m}, 2 \mathrm{H}), 2.37(\mathrm{t}, J=7.6 \mathrm{~Hz}, 2 \mathrm{H}), 5.44(\mathrm{br} \mathrm{s}, 2 \mathrm{H}), 8.08(\mathrm{~s}, 2 \mathrm{H}) ;{ }^{13} \mathrm{C}$ NMR $\delta 13.9$, 22.3, 29.4, 30.7, 31.0, 124.6, 157.8, 161.8; FAB-MS $m / z 166\left(\left[\mathrm{M}+\mathrm{H}^{+}\right], 100 \%\right)$; HRMS $m / z$ $166.1339\left(\mathrm{M}+\mathrm{H}^{+}\left[\mathrm{C}_{9} \mathrm{H}_{16} \mathrm{~N}_{3}\right]=166.1344\right)$. 
(i) 2-Amino-5-(hex-1-yn-1-yl)pyrimidine: ${ }^{1} \mathrm{H}$ NMR $\delta 0.95(\mathrm{t}, J=7.3 \mathrm{~Hz}, 3 \mathrm{H}), 1.42-1.62(\mathrm{~m}$, 4H), $2.40(\mathrm{t}, J=7.3 \mathrm{~Hz}, 2 \mathrm{H}), 5.30($ br s, $2 \mathrm{H}), 8.31(\mathrm{~s}, 2 \mathrm{H}) ;{ }^{13} \mathrm{C}$ NMR $\delta 13.5,19.1,22.0,30.7 .1$, 74.5, 93.5, 109.5, 160.3, 161.0; FAB-MS m/z $176\left(\left[\mathrm{M}+\mathrm{H}^{+}\right], 100 \%\right) ;$ HRMS m/z 176.1179 (M + $\mathrm{H}^{+}\left[\mathrm{C}_{10} \mathrm{H}_{14} \mathrm{~N}_{3}\right]=176.1088$ ). (ii) Hydrogenation of this material gave 2-amino-5hexylpyrimidine (22b). (iii) Benzyltriethylammonium nitrite ${ }^{6}\left(\mathrm{BTEANO}_{2}\right)(2.60 \mathrm{~g}, 10.9 \mathrm{mmol})$ in $\mathrm{CH}_{2} \mathrm{Cl}_{2}(20 \mathrm{~mL})$ was added to a stirred solution of $\mathbf{2 2 b}(600 \mathrm{mg}, 3.35 \mathrm{mmol})$ and TMSCl (4.28 $\mathrm{mL}, 3.67 \mathrm{~g}, 33.8 \mathrm{mmol})$ in $\mathrm{CH}_{2} \mathrm{Cl}_{2}(16 \mathrm{~mL})$. Stirring was continued for $3 \mathrm{~h}$ at ambient temperature, and volatiles were evaporated. The residue was chromatographed $\left(\mathrm{CH}_{2} \mathrm{Cl}_{2}\right)$ to give 2-chloro-5-hexylpyrimidine (23b) $(450 \mathrm{mg}, 68 \%):{ }^{1} \mathrm{H}$ NMR $\delta 0.89(\mathrm{t}, J=6.8 \mathrm{~Hz}, 3 \mathrm{H})$, $1.27-1.38(\mathrm{~m}, 6 \mathrm{H}), 1.59-1.65(\mathrm{~m}, 2 \mathrm{H}), 2.60(\mathrm{t}, J=7.8 \mathrm{~Hz}, 2 \mathrm{H}), 8.45(\mathrm{~s}, 2 \mathrm{H}) ;{ }^{13} \mathrm{C}$ NMR $\delta 13.6$, 22.1, 28.2, 29.1, 30.1, 31.0, 133.8, 158.6, 158.9; FAB-MS m/z $199\left(\left[\mathrm{M}+\mathrm{H}^{+}\right], 100 \%\right)$; HRMS $m / z 199.1003\left(\mathrm{M}+\mathrm{H}^{+}\left[\mathrm{C}_{10} \mathrm{H}_{16} \mathrm{ClN}_{2}\right]=199.1002\right)$.

General Procedures for Synthesis of 24 and 25. (i) Sonogashira couplings of 23 and TMSacetylene followed by desilylation were performed as described for conversions of the 5-alkyl-2bromopyridines $\rightarrow$ 13. (ii) Sonogashira couplings of the resulting 5-alkyl-2-ethynylpyrimidines with 1-[2-deoxy-3,5-di-O-(p-toluoyl)- $\beta$-D-erythro-pentofuranosyl]-5-iodouracil were followed by (iii) removal of the $p$-toluoyl groups and (iv) cyclization (as described for $\mathbf{1 3} \rightarrow \mathbf{1 4} \rightarrow \mathbf{1 5}$ ).

\section{1-[2-Deoxy-3,5-di-O-(p-toluoyl)- $\beta$-D-erythro-pentofuranosyl]-5-[2-(5-pentylpyrimid-2-}

yl)ethynyl]uracil (24a). ${ }^{1} \mathrm{H}$ NMR $\delta 0.91(\mathrm{t}, J=7.3 \mathrm{~Hz}, 3 \mathrm{H}), 1.31-1.38(\mathrm{~m}, 4 \mathrm{H}), 1.60-1.67(\mathrm{~m}$, $2 \mathrm{H}), 2.29,2.43(2 \times \mathrm{s}, 2 \times 3 \mathrm{H}), 2.26-2.32(\mathrm{~m}, 1 \mathrm{H}), 2.60(\mathrm{t}, J=7.8 \mathrm{~Hz}, 2 \mathrm{H}), 2.77(\mathrm{ddd}, J=1.5$, $5.4,14.2 \mathrm{~Hz}, 1 \mathrm{H}), 4.56-4.59(\mathrm{~m}, 1 \mathrm{H}), 4.65,4.82(2 \times \mathrm{dd}, J=3.4,12.2 \mathrm{~Hz}, 2 \times 1 \mathrm{H}), 5.59(\mathrm{~d}, J=$ $6.3 \mathrm{~Hz}, 1 \mathrm{H}), 6.35(\mathrm{dd}, J=5.4,8.3 \mathrm{~Hz}, 1 \mathrm{H}), 7.18,7.27,7.91,7.93(4 \times \mathrm{d}, J=8.3 \mathrm{~Hz}, 4 \times 2 \mathrm{H})$, 7.26, 8.13, $8.54(3 \times \mathrm{s}, 3 \times 1 \mathrm{H}), 8.39($ br s, $1 \mathrm{H}) ;{ }^{13} \mathrm{C}$ NMR $\delta 13.8,21.5,21.6,22.2,30.1,30.2$, 
$31.1,38.2,64.0,74.9,78.7,83.2,86.0,91.7,99.3,126.3,126.5,129.1,129.2,129.6,129.7$, 134.1, 143.9, 144.0, 144.3, 149.3, 150.3, 156.8, 160.7, 165.7, 166.1; FAB-MS m/z $637([\mathrm{M}+$ $\left.\left.\mathrm{H}^{+}\right], 70 \%\right), 285(100 \%)$; HRMS $m / z 637.2657\left(\mathrm{M}+\mathrm{H}^{+}\left[\mathrm{C}_{36} \mathrm{H}_{37} \mathrm{~N}_{4} \mathrm{O}_{7}\right]=637.2662\right)$.

3-(2-Deoxy-ß-D-erythro-pentofuranosyl)-6-(2-pentylpyrimid-5-yl)furo[2,3-d]pyrimidin2(3H)-one (25a). ${ }^{1} \mathrm{H}$ NMR (DMSO- $\left.d_{6}\right) \delta 0.88(\mathrm{t}, J=7.3 \mathrm{~Hz}, 3 \mathrm{H}), 1.26-1.36(\mathrm{~m}, 4 \mathrm{H}), 1.59-1.66$ (m, 2H), $2.12(\mathrm{td}, J=5.8,13.7 \mathrm{~Hz}, 1 \mathrm{H}), 2.45(\mathrm{ddd}, J=4.4,5.9,13.7 \mathrm{~Hz}, 1 \mathrm{H}), 2.50-2.54(\mathrm{~m}$, $1 \mathrm{H}), 2.64(\mathrm{t}, J=7.8 \mathrm{~Hz}, 2 \mathrm{H}), 3.62-3.74(\mathrm{~m}, 2 \mathrm{H}), 3.94-3.97(\mathrm{~m}, 1 \mathrm{H}), 4.23-4.27(\mathrm{~m}, 1 \mathrm{H}), 5.13(\mathrm{t}$, $J=5.1 \mathrm{~Hz}, 1 \mathrm{H}), 5.30(\mathrm{~d}, J=3.9 \mathrm{~Hz}, 1 \mathrm{H}), 6.17(\mathrm{t}, J=6.1 \mathrm{~Hz}, 1 \mathrm{H}), 7.51,8.99(2 \times \mathrm{s}, 2 \times 1 \mathrm{H})$, $8.79(\mathrm{~s}, 2 \mathrm{H}) ;{ }^{13} \mathrm{C}$ NMR $\left(\mathrm{DMSO}-d_{6}\right) \delta$ 13.8, 21.8, 29.3, 29.7, 30.7, 41.2, 60.8, 69.7, 88.0, 88.4, $105.9,106.5,134.4,140.6,151.6,153.8,154.1,157.3,171.2 ;$ FAB-MS $m / z 423\left(\left[\mathrm{M}+\mathrm{Na}^{+}\right]\right.$, 60\%), $285(100 \%)$; HRMS $m / z, 423.1648\left(\mathrm{M}+\mathrm{Na}^{+}\left[\mathrm{C}_{20} \mathrm{H}_{24} \mathrm{~N}_{4} \mathrm{O}_{5} \mathrm{Na}\right]=423.1639\right)$.

\section{1-[2-Deoxy-3,5-di-O-(p-toluyl)- $\beta$-D-erythro-pentofuranosyl]-5-[2-(5-hexylpyrimid-2-}

yl)ethynyl]uracil (24b). ${ }^{1} \mathrm{H}$ NMR $\delta 0.89(\mathrm{t}, J=7.3 \mathrm{~Hz}, 3 \mathrm{H}), 1.28-1.38(\mathrm{~m}, 6 \mathrm{H}), 1.60-1.66(\mathrm{~m}$, 2H), 2.29, $2.42(2 \times \mathrm{s}, 2 \times 3 \mathrm{H}), 2.38(\mathrm{td}, J=6.1,13.2 \mathrm{~Hz}, 1 \mathrm{H}), 2.62(\mathrm{t}, J=7.8 \mathrm{~Hz}, 2 \mathrm{H}), 2.79$ $(\mathrm{ddd}, J=1.5,7.8,14.2 \mathrm{~Hz}, 1 \mathrm{H}), 4.56-4.59(\mathrm{~m}, 1 \mathrm{H}), 4.65,4.80(2 \times \mathrm{dd}, J=3.4,12.2 \mathrm{~Hz}, 2 \times$ $1 \mathrm{H}), 5.59(\mathrm{~d}, J=6.4 \mathrm{~Hz}, 1 \mathrm{H}), 6.34(\mathrm{dd}, J=5.4,8.3 \mathrm{~Hz}, 1 \mathrm{H}), 7.17,7.26,7.91,7.93(4 \times \mathrm{d}, J=8.3$ $\mathrm{Hz}, 4 \times 2 \mathrm{H}), 7.26,8.20,8.61(3 \times \mathrm{s}, 3 \times 1 \mathrm{H}), 9.09(\mathrm{br} \mathrm{s}, 1 \mathrm{H}) ;{ }^{13} \mathrm{C}$ NMR $\delta 13.9,21.4,21.5,22.4$ 28.6, 30.2, 30.3, 31.3, 38.0, 64.0, 74.9, 79.1, 83.1, 85.9, 91.5, 99.1, 126.3, 126.4, 129.06, 129.14, 129.5, 129.7, 134.1, 143.8, 144.2, 144.3, 149.4, 150.1, 156.8, 160.9, 165.7, 166.0; FAB-MS m/z $651\left(\left[\mathrm{M}+\mathrm{H}^{+}\right], 100 \%\right) ; \mathrm{HRMS} m / z 651.2829\left(\mathrm{M}+\mathrm{H}^{+}\left[\mathrm{C}_{37} \mathrm{H}_{39} \mathrm{~N}_{4} \mathrm{O}_{7}\right]=651.2813\right)$.

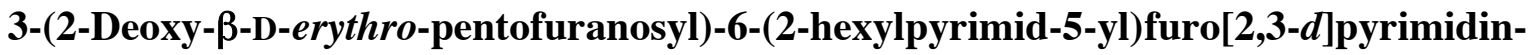

2(3H)-one (25b). ${ }^{1} \mathrm{H}$ NMR (DMSO- $\left.d_{6}\right) \delta 0.86(\mathrm{t}, J=7.3 \mathrm{~Hz}, 3 \mathrm{H}), 1.24-1.35(\mathrm{~m}, 6 \mathrm{H}), 1.58-1.65$ (m, 2H), $2.12(\mathrm{td}, J=6.1,13.7 \mathrm{~Hz}, 1 \mathrm{H}), 2.44(\mathrm{ddd}, J=4.4,5.9,13.7 \mathrm{~Hz}, 1 \mathrm{H}), 2.50-2.53(\mathrm{~m}$, 
1H), $2.64(\mathrm{t}, J=7.8 \mathrm{~Hz}, 2 \mathrm{H}), 3.62-3.73(\mathrm{~m}, 2 \mathrm{H}), 3.95-4.00(\mathrm{~m}, 1 \mathrm{H}), 4.23-4.27(\mathrm{~m}, 1 \mathrm{H}), 5.13(\mathrm{t}$, $J=5.1 \mathrm{~Hz}, 1 \mathrm{H}), 5.30(\mathrm{~d}, J=4.4 \mathrm{~Hz}, 1 \mathrm{H}), 6.17(\mathrm{t}, J=6.1 \mathrm{~Hz}, 1 \mathrm{H}), 7.50,8.99(2 \times \mathrm{s}, 2 \times 1 \mathrm{H})$, $8.78(\mathrm{~s}, 2 \mathrm{H}) ;{ }^{13} \mathrm{C}$ NMR (DMSO- $\left.d_{6}\right) \delta 13.9,22.0,28.2,29.4,30.0,30.9,41.2,60.8,69.7,88.0$, $88.4,105.9,106.5,134.4,140.6,151.6,153.8,154.1,157.3,171.2 ;$ FAB-MS m/z $437([\mathrm{M}+$ $\left.\left.\mathrm{Na}^{+}\right], 30 \%\right), 299$ (100\%); HRMS m/z $437.1795\left(\mathrm{M}+\mathrm{Na}^{+}\left[\mathrm{C}_{21} \mathrm{H}_{26} \mathrm{~N}_{4} \mathrm{O}_{5} \mathrm{Na}\right]=437.1798\right)$. 
Elemental Analyses within $\pm 0.4 \%$ of Theory

\begin{tabular}{|l|c|c|c|c|c|c|}
\hline \multirow{2}{*}{ Compd } & \multicolumn{3}{|c|}{ Calcd } & \multicolumn{3}{c|}{ Found } \\
\cline { 2 - 7 } & $\mathrm{C}$ & $\mathrm{H}$ & $\mathrm{N}$ & $\mathrm{C}$ & $\mathrm{H}$ & $\mathrm{N}$ \\
\hline 9b(i) & 64.77 & 4.58 & 7.95 & 64.57 & 4.71 & 7.69 \\
\hline 9b(iv) & 68.23 & 6.20 & 6.63 & 68.46 & 6.36 & 6.64 \\
\hline 10b(i) & 64.04 & 5.66 & 7.86 & 63.68 & 5.88 & 7.59 \\
\hline 10b(ii) & 67.59 & 7.09 & 6.57 & 67.40 & 6.96 & 6.52 \\
\hline 10b(iii) & 66.32 & 6.58 & 7.03 & 66.16 & 6.76 & 6.93 \\
\hline 10b(iv) & 67.59 & 7.09 & 6.57 & 67.40 & 6.96 & 6.52 \\
\hline
\end{tabular}



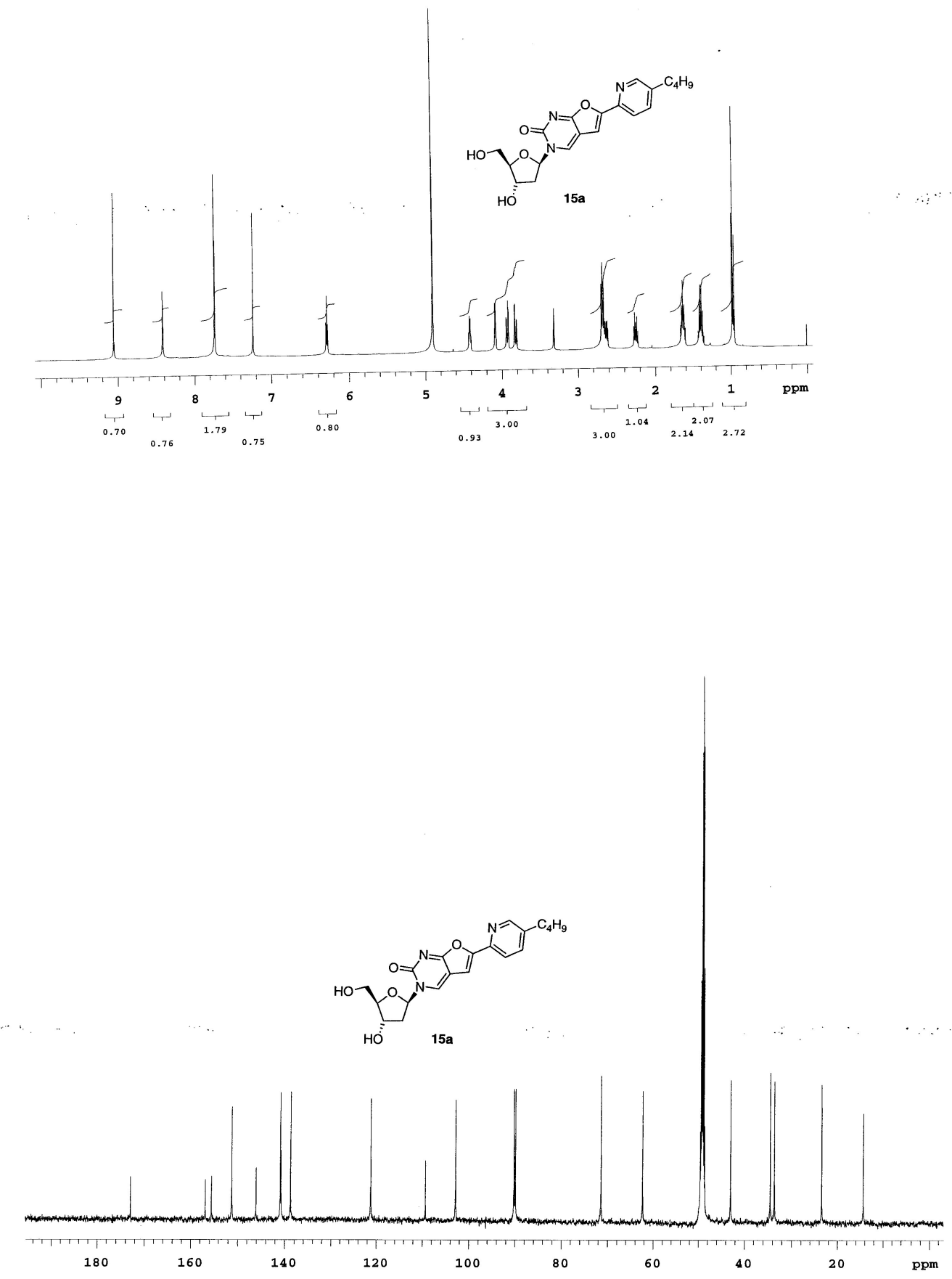

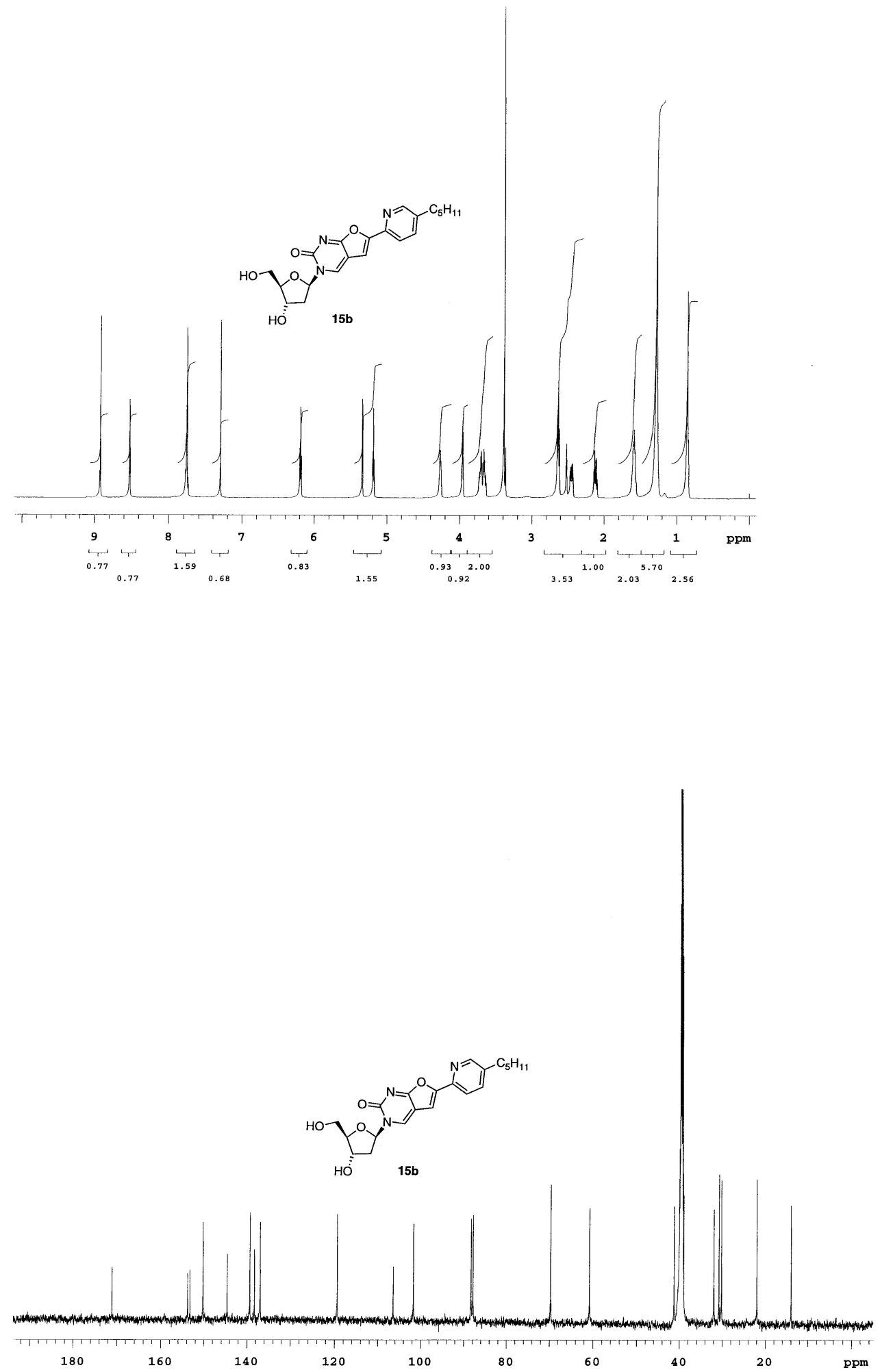

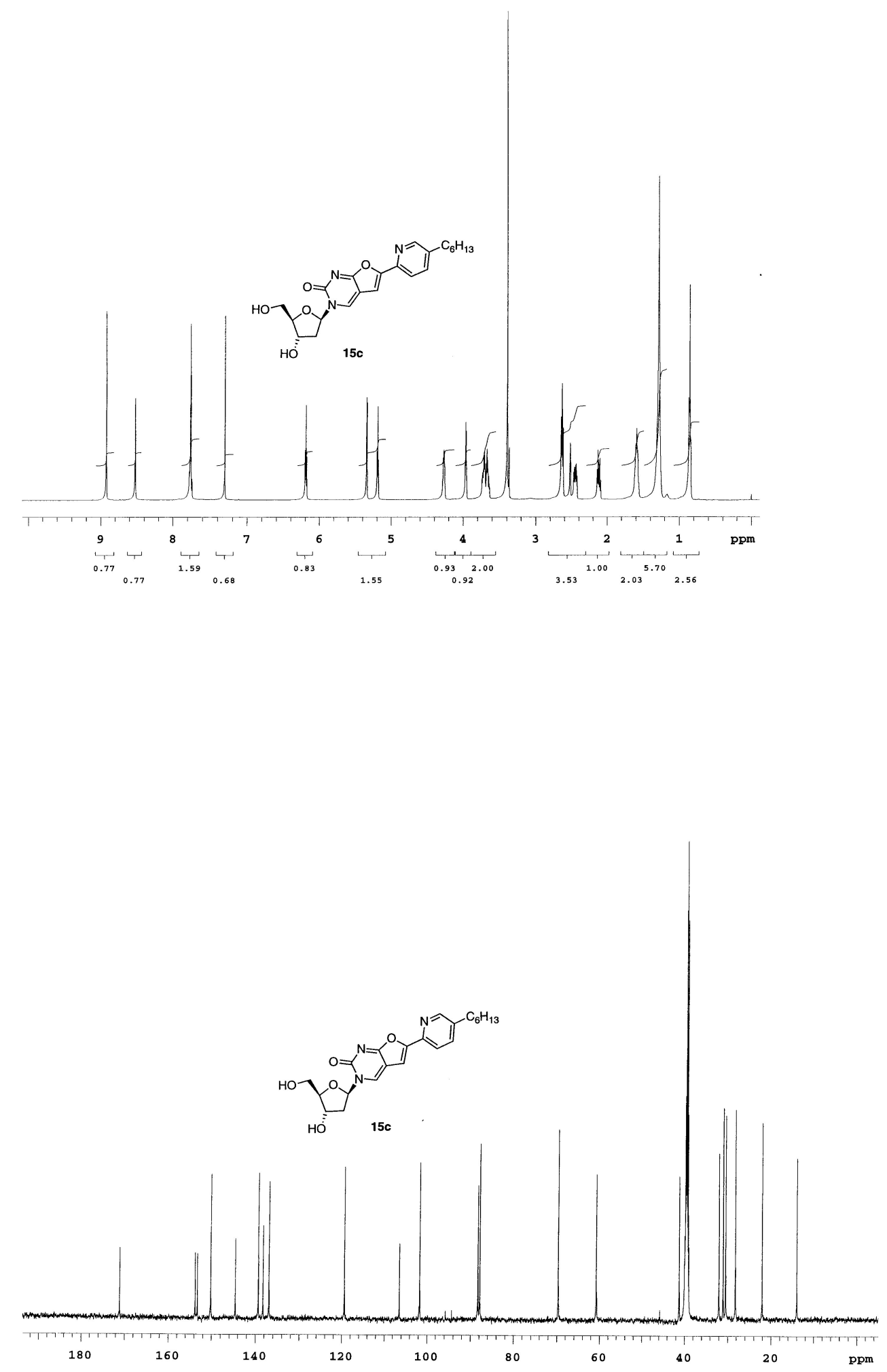

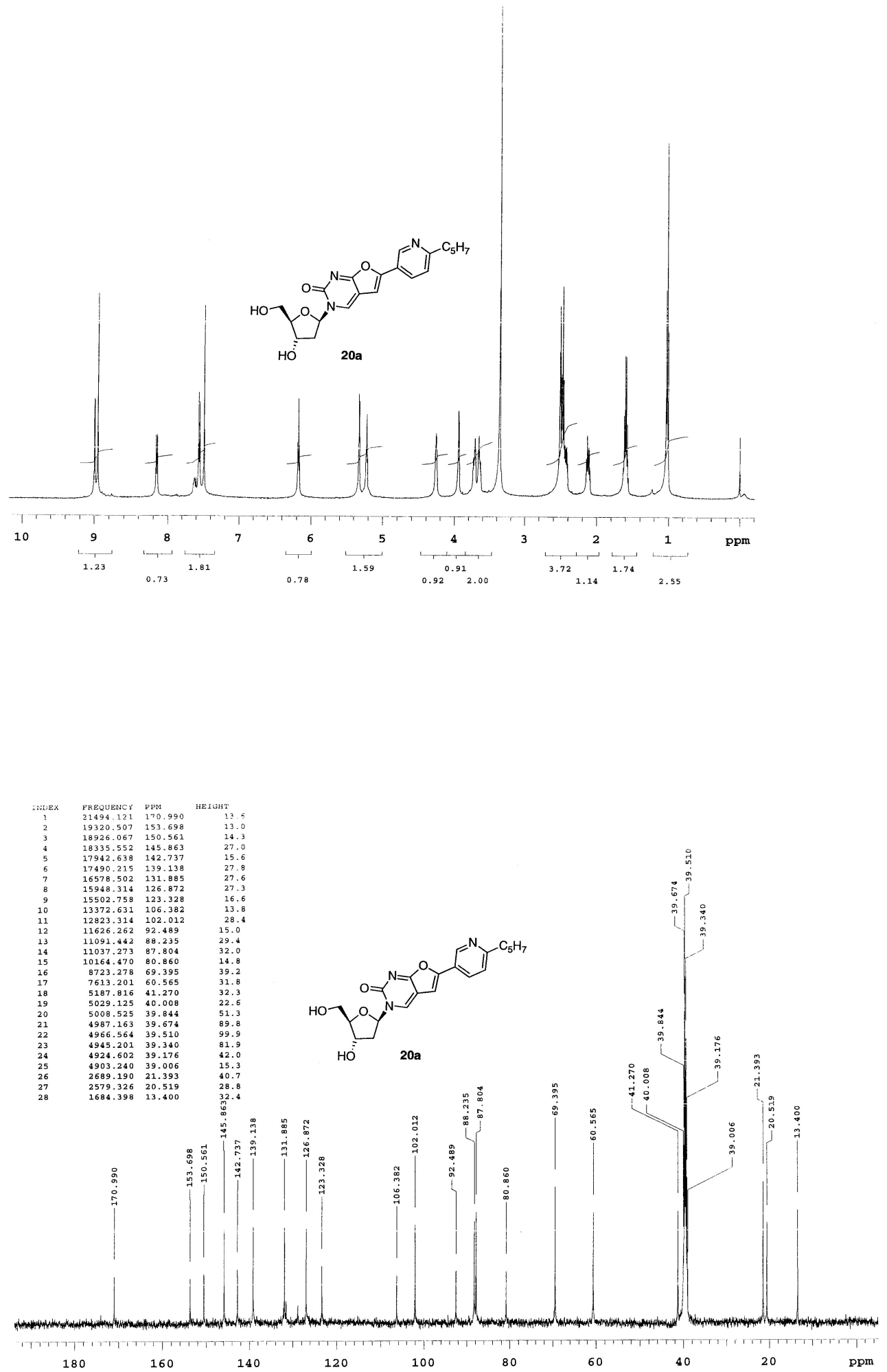


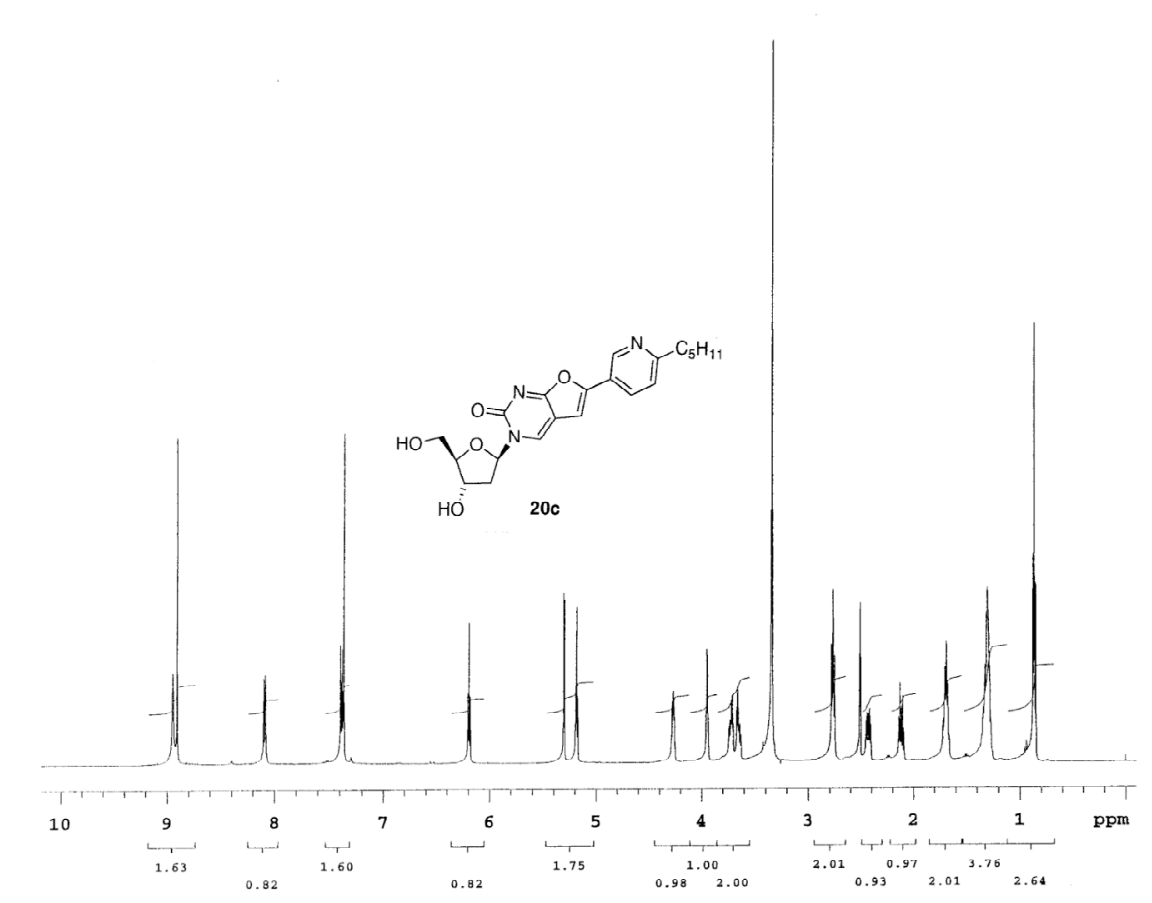

I

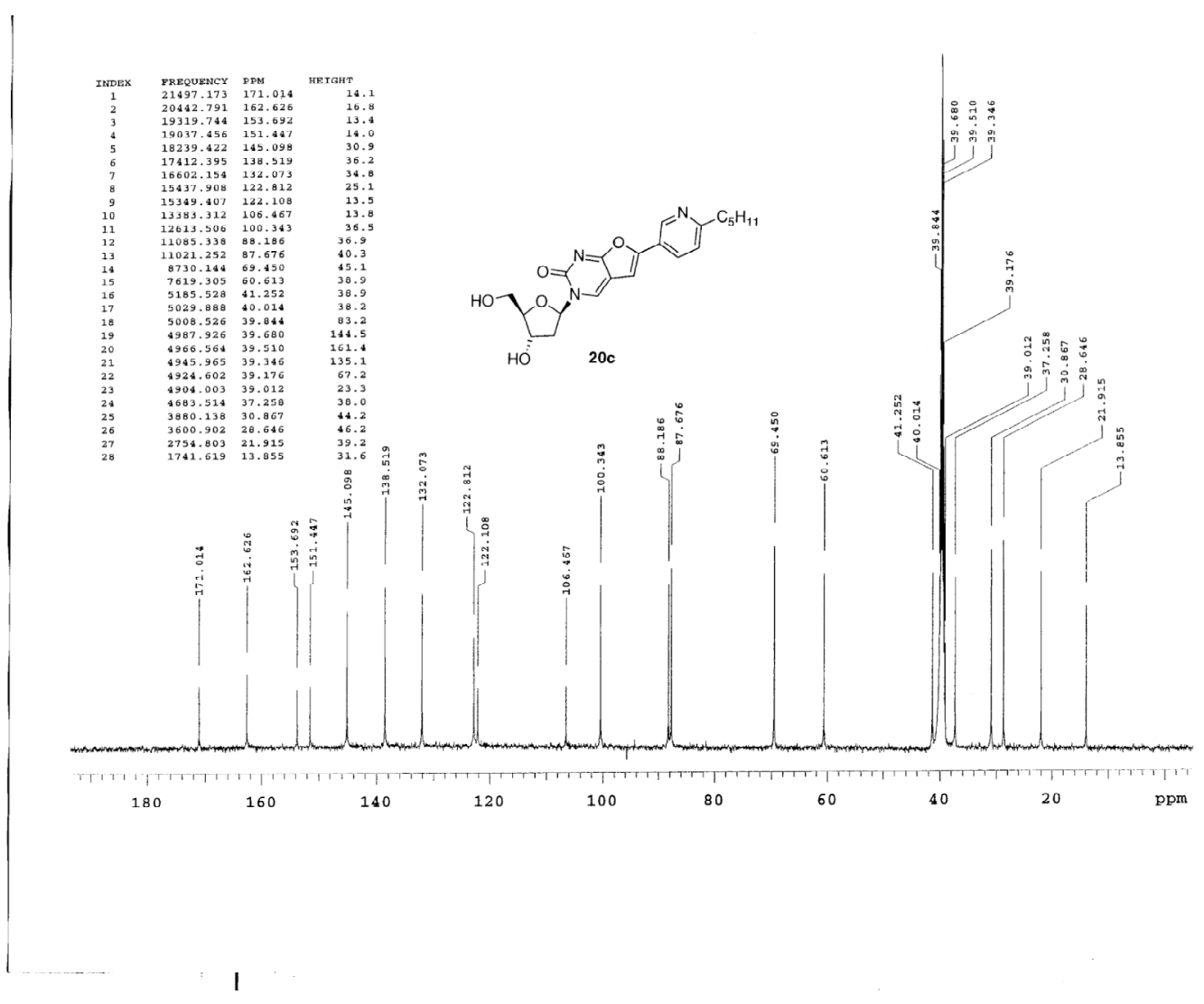




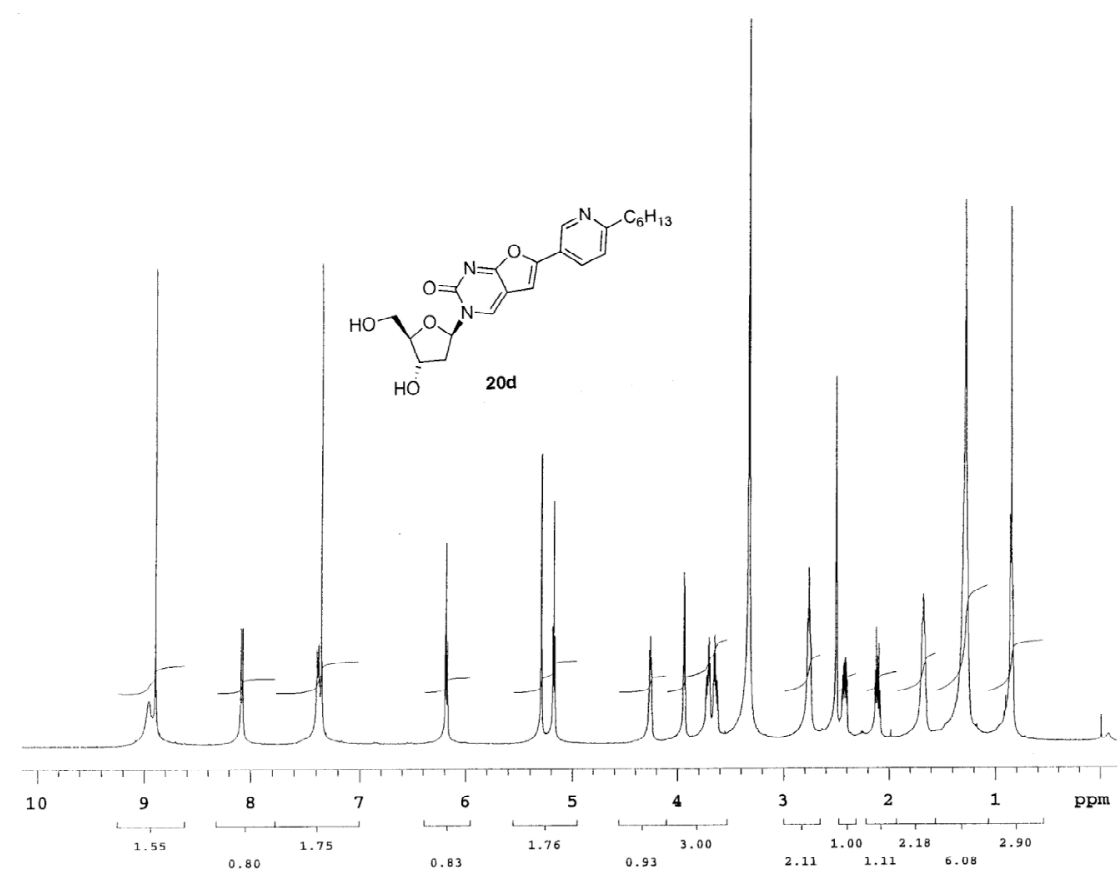

I

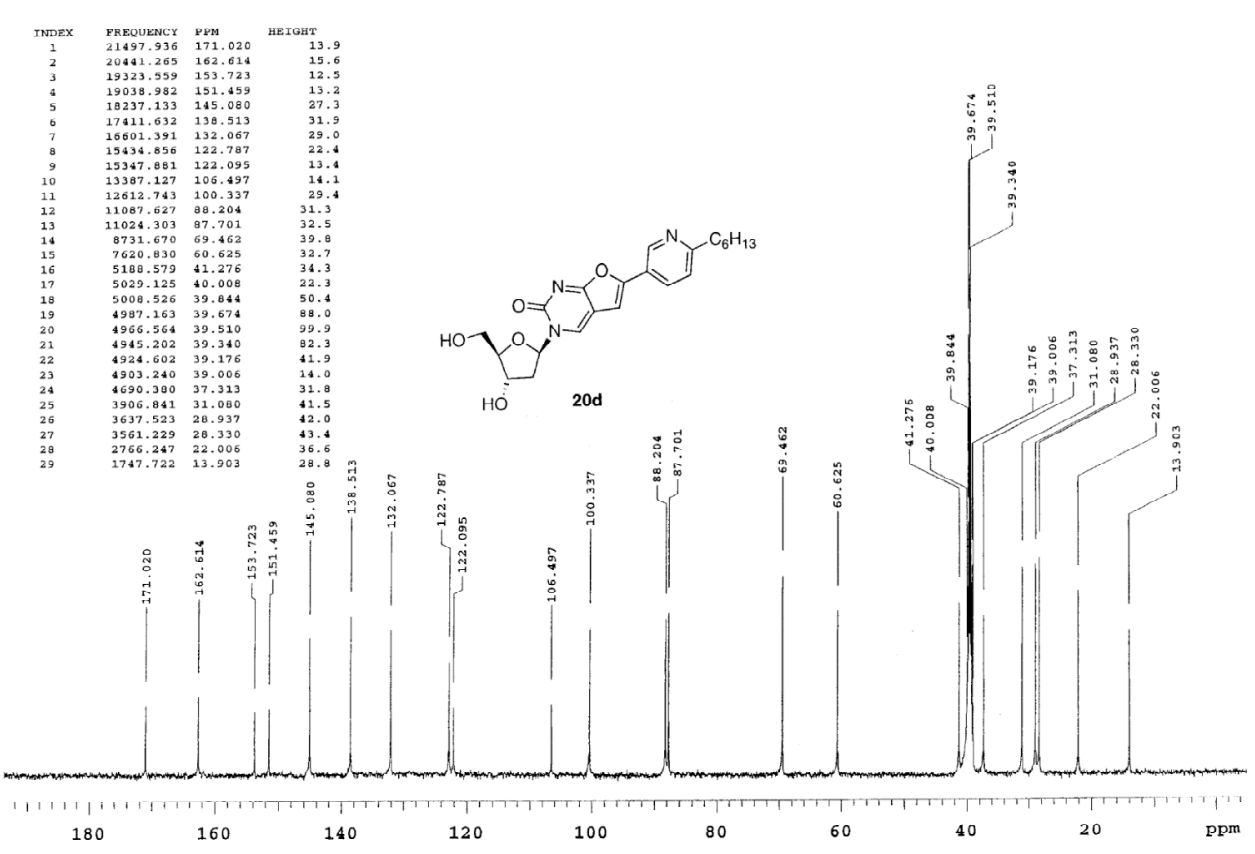



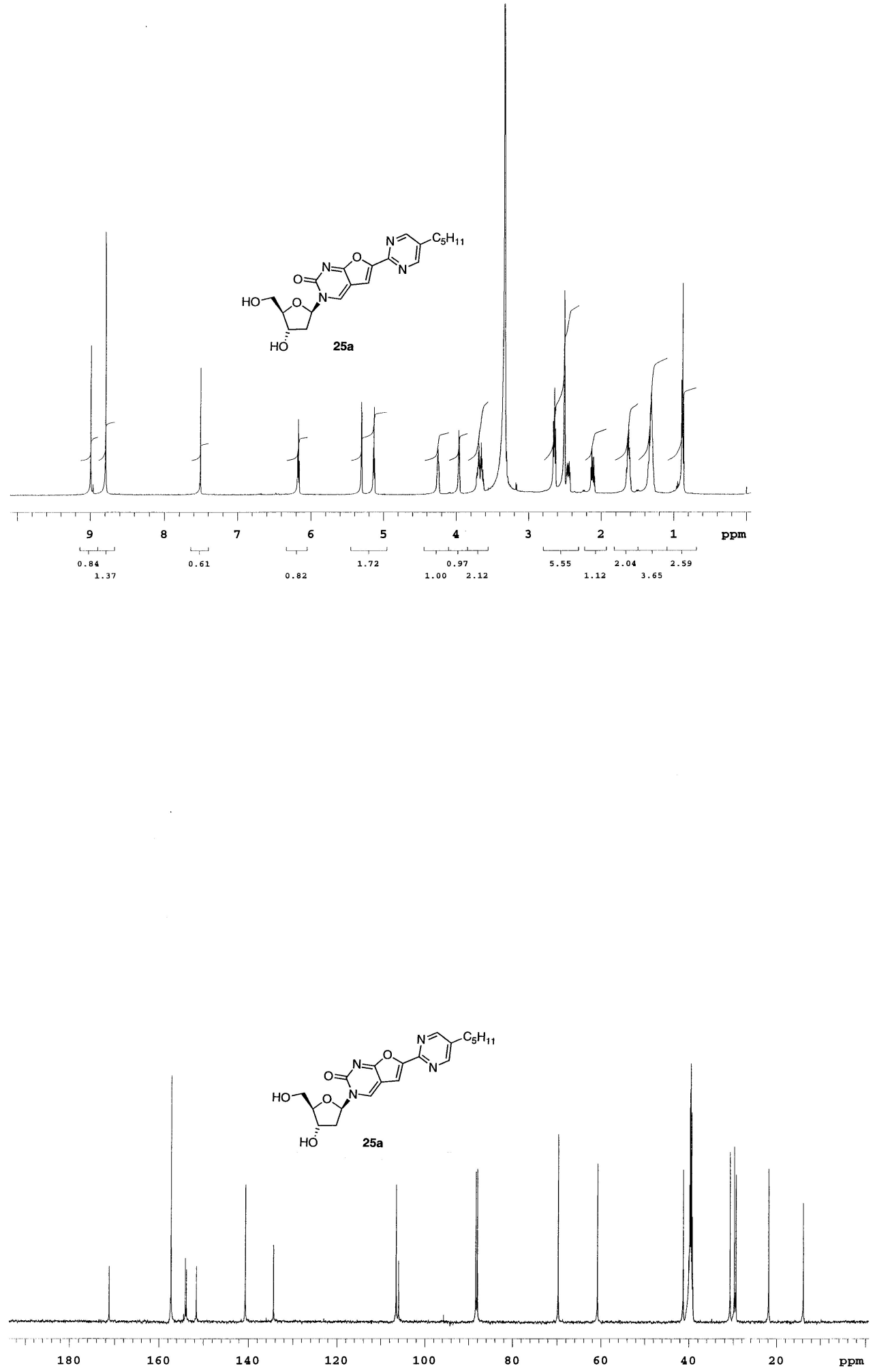

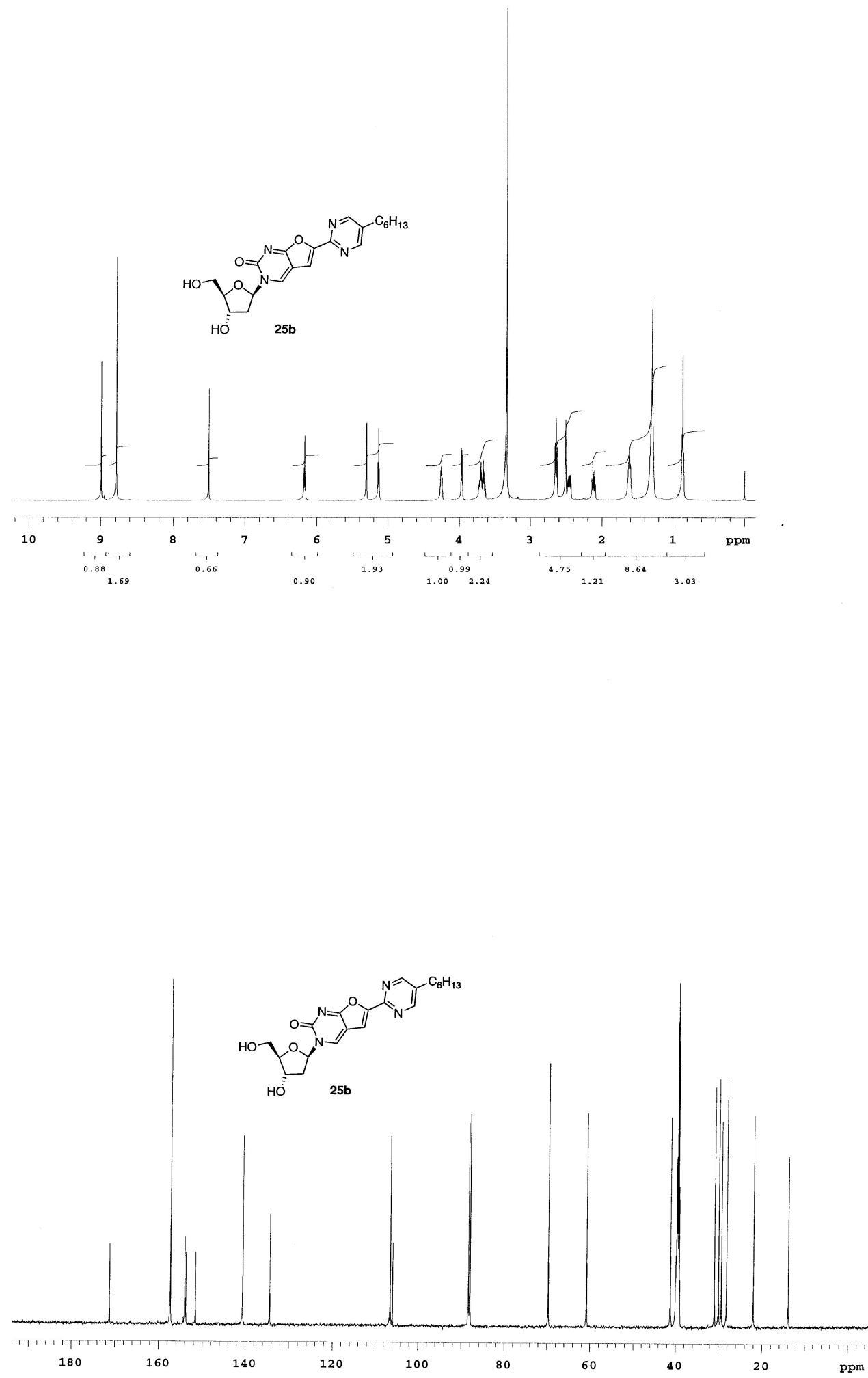
${ }^{1}$ Robins, M. J.; Miranda, K.; Rajwanshi, V. K.; Peterson, M. A.; Andrei, G.; Snoeck, R.; De Clercq, E.; Balzarini, J. Synthesis and Biological Evaluation of 6-(Alkyn-1-yl)furo[2,3d]pyrimidin-2(3H)-one Base and Nucleoside Derivatives. J. Med. Chem. 2006, 49, 391-398.

${ }^{2}$ McGuigan, C.; Yarnold, C. J.; Jones, G.; Velazquez, S.; Barucki, H.; Brancale, A.; Andrei, G.; Snoeck, R.; De Clercq, E.; Balzarini, J. Potent and Selective Inhibition of Varicella-Zoster Virus (VZV) by Nucleoside Analogues with an Unusual Bicyclic Base. J. Med. Chem. 1999, 42, 4479-4484.

${ }^{3}$ Mathieu, J.; Gros, P.; Fort, Y. Unprecedented C-6 Functionalisation of 3-Picosine Induced by a Methyl to C-6 Lithium Shift. J. Chem. Soc., Chem. Commun. 2000, 951-952.

${ }^{4}$ Schwab, P. F. H.; Fleischer, F.; Michl, J. Preparation of 5-Brominated and 5,5'-Dibrominated 2,2'-Bipyridines and 2,2'-Bipyrimidines. J. Org. Chem. 2002, 67, 443-449.

${ }^{5}$ Tilley, J. W., Zawoiski, S. A Convenient Palladium-Catalyzed Coupling Approach to 2,5Disubstituted Pyridines. J. Org. Chem. 1988, 53, 386-390.

${ }^{6}$ (a) Francom, P.; Janeba, Z.; Shibuya, S.; Robins, M. J. Nucleic Acid Related Compounds. 116. Nonaqueous Diazotization of Aminopurine Nucleosides. Mechanistic Considerations and Efficient Procedures with tert-Butyl Nitrite or Sodium Nitrite. J. Org. Chem. 2002, 67, 6788-6796. (b) Francom, P.; Robins, M. J. Nucleic Acid Related Compounds. 118. Nonaqueous Diazotization of Aminopurine Derivatives. Convenient Access to 6-Halo- and 2,6-Dihalopurine Nucleosides with Acyl or Silyl Halides. J. Org. Chem. 2003, 68, 666-669. 